\title{
Sequencing ultra-long DNA molecules with the Oxford Nanopore MinION
}

\author{
John M. Urban ${ }^{1,3^{*}}$, Jacob Bliss ${ }^{1}$, Charles E. Lawrence ${ }^{2,3}$, Susan A. Gerbi ${ }^{1,3^{*}}$
}

${ }^{1}$ BioMed Division (MCB Department), Brown University, Providence, RI, USA

${ }^{2}$ Division of Applied Mathematics, Brown University, Providence, RI, USA

${ }^{3}$ Center for Computational Molecular Biology, Brown University, Providence, RI, USA

*Correspondence to John_Urban@Brown.edu and Susan_Gerbi@Brown.edu

Key Words: Oxford Nanopore MinION; DNA sequencing; nanopore sequencing; long reads; long DNA preparation 


\begin{abstract}
Oxford Nanopore Technologies' nanopore sequencing device, the MinION, holds the promise of sequencing ultra-long DNA fragments $>100 \mathrm{~kb}$. An obstacle to realizing this promise is delivering ultra-long DNA molecules to the nanopores. We present our progress in developing cost-effective ways to overcome this obstacle and our resulting MinION data, including multiple reads $>100 \mathrm{~kb}$.
\end{abstract}


High-throughput DNA sequencing is at the cusp of two paradigm shifts: (1) where and (2) how sequencing is performed. The MinION from Oxford Nanopore Technologies (ONT) is a pocket-sized, long-read $(>1 \mathrm{~kb})$ DNA sequencing device used in individual laboratories and fieldwork ${ }^{1-4}$, presently limited to participants in the MinION Access Programme (MAP), that detects 5mer dependent changes in ionic current, or "events" (consisting of a mean, standard deviation, start time, and duration), as single DNA molecules traverse nanopores. The events are then base-called using ONT's Metrichor cloud service. There has been a trend of increasing base-calling accuracy and total output per flow cell ${ }^{5-9}$, now up to $85 \%{ }^{9}$ and $490 \mathrm{Mb}^{7}$, with expectations of soon exceeding $90 \%$ accuracy and $2 \mathrm{~Gb}$ per flow cell. Importantly, since MinION reads can be re-base-called, older reads can inherit the benefits of better base-calling.

The protocol for preparing a MinION sequencing library is still evolving, but currently includes shearing genomic DNA using a Covaris g-TUBE, an optional "PreCR" step to repair damaged DNA, end-repair, dA-tailing, adapter ligation, and His-bead purification. When properly ligated, a double-stranded (ds) DNA molecule has a Yshaped $(\mathrm{Y})$ adapter at one end and a hairpin (HP) adapter at the other. dsDNA is pulled through a pore one strand at a time starting at the 5' end of the $\mathrm{Y}$, followed by the "template" strand, and, ideally, the HP and "complement" strand. The information from either strand can be used for 1-directional (1D) base-calling, and integrating the information from both strands can be used for 2-directional (2D) base-calling, which results in higher mean quality scores (Q; Supplementary Table 1) and higher accuracy ${ }^{6-}$ 11. Molecules with a $\mathrm{Y}$ at each end or a nick in the template only give 1D template reads. It is also possible to obtain information from both strands but have no 2D base-calling (Supplementary Fig. 1, Supplementary Table 2). There is an approximate ratio of 1 
event per base in 1D reads and 2 events per base in 2D reads (Supplementary Fig. 2). 2D reads with mean quality scores $Q \geq 9$ are considered high quality, although most other $2 \mathrm{D}$ reads $\left(83-91 \%\right.$ align to a reference ${ }^{8,11}$ ) and many $1 \mathrm{D}$ reads (e.g., $\mathrm{Q} \geq 3.5$; Supplementary Fig. 2) are also of useful quality for various applications ${ }^{2,12-17}$, especially if reads are first error-corrected ${ }^{7,11,16,18}$ or if one does not propagate the error from basecalling by working directly with the events instead ${ }^{16,18}$.

The MinION has the promise to sequence ultra-long DNA fragments $>100 \mathrm{~kb}^{19}$. However, early reports suggested it falls short of this promise ${ }^{5,20}$ with maximum read lengths near $10 \mathrm{~kb}$. In more recent reports, the majority of reads were concentrated below $30 \mathrm{~kb}$, but maximum read lengths were approximately $31.6 \mathrm{~kb}(2 \mathrm{D})^{8}, 48.5 \mathrm{~kb}(2 \mathrm{D})^{9}$, $58.7 \mathrm{~kb}(2 \mathrm{D})^{11}, 66.7 \mathrm{~kb}(1 \mathrm{D})^{8}, 123 \mathrm{~kb}(1 \mathrm{D})^{11}$, and $147 \mathrm{~kb}(1 \mathrm{D})^{7}$. Nonetheless, reads $>100 \mathrm{~kb}$ have been rare and no instances of $2 \mathrm{D}$ reads $>100 \mathrm{~kb}$ have yet been reported. Here we describe modified protocols to harness the MinION's potential for sequencing ultra-long molecules and present three resulting MinION runs (A, B, and $C$ ) with multiple reads exceeding 100kb (Fig. 1, Table 1, Supplementary Table 3).

First, for Run A, we sought to maximize read lengths by gently (no vortexing) letting freshly obtained (never frozen) precipitated DNA re-suspend in TE (pH 8.0), skipping the Covaris shearing step, using wide-bored tips with gentle pipetting to minimize DNA breakage, and starting with $3 X$ the recommended starting material to compensate for differences in molarity (Supplementary Fig. 3). Moreover, in all AMPure clean-ups we performed long elutions (20 minutes) at $37^{\circ} \mathrm{C}$ to help release long DNA from the beads. Run A had 9935 base-called events files featuring 139 molecules $>50 \mathrm{~kb}, 21$ molecules $>100 \mathrm{~kb}$, a max 2D length of 102.935kb $(\mathrm{Q}=8.74)$ and a max 1D 
length of $304.309 \mathrm{~kb}(Q=2.12)$, although the longest $1 \mathrm{D}$ length with $Q>3.5$ was 202.293kb (Fig. 1, Table 1). The summed non-redundant molecule length was 49.8Mb with a molecule N50 of $25.238 \mathrm{~kb}$ (Table 1). $79.2 \%$ of the summed molecule length came from molecules greater than $10 \mathrm{~kb}$, which made up $14.4 \%$ of all molecules sequenced (Table 1).

There were three side effects when keeping the DNA long (Table 1): (1) a low proportion of 2D reads (only $\sim 21.9 \%$ of base-called molecules had a 2D read); (2) a high number of tiny events files $(87.2 \%$ of pre-base-called events files contained fewer than 2000 events although this made up only $3.41 \%$ of all events obtained); and (3) lower output than might have been achieved if the DNA was sheared to shorter lengths (100-400Mb routinely achieved by others in MAP at the time of our experiments). One possible explanation is that ultra-long DNA is fragile and therefore can break after endrepair leading to problems in ligation, can break after ligation leading to His-bead enrichments of HP-ligated DNA that cannot be sequenced, and can break while being injected into the MinION leading to more issues such as Y-ligated DNA that can only give 1D reads. Therefore, we proceeded to find a balance between read length, total output, and proportion of 2D reads.

For Run B we sought to minimize post-repair breaks while keeping a large proportion of the DNA $>10 \mathrm{~kb}$ (Supplementary Fig. 3). To do so we vortexed the DNA (full speed, 30 seconds) after DNA extraction (Supplementary Fig. 4A) and used normal pipette tips until end-repair, after which wide-bored tips and gentler pipetting were employed. Moreover, to compensate for the possible increase of molecules $<1 \mathrm{~kb}$ due to vortexing, two sequential 0.4x AMPure bead clean-ups were performed after end-repair. 
Run B had a bigger proportion of files with 2D reads (49.8\%), a smaller proportion of tiny events files $(40.2 \%$ of events files had $<2000$ events making up $2.48 \%$ of all events), and a much higher output (386.9Mb of non-redundant molecules) albeit with a lower yet still impressive molecule N50 of 13.553kb (Table 1). Run B had proportionally fewer reads $>50 \mathrm{~kb}$ than Run $A$, but there were more in terms of total count owing to the higher output (396 molecules $>50 \mathrm{~kb}, 24>100 \mathrm{~kb}$ ). The longest 2D read was $86.8 \mathrm{~kb}$ $(Q=9.01)$ and the longest $1 D$ read was $671.219 \mathrm{~kb}(Q=1.55)$, but the longest $1 D$ read with $Q>3.5$ was $143.763 \mathrm{~kb}$. The majority (58.5\%) of the summed molecule length came from molecules $>10 \mathrm{~kb}$ ( $15.8 \%$ of all base-called molecules).

For Run C, we sought to improve upon Run B by increasing the amount of data from molecules $>10 \mathrm{~kb}$. We first explored ways to deplete DNA molecules smaller than $10 \mathrm{~kb}$ with simple modifications to the standard AMPure bead protocol (Supplementary Fig. 4B) and found that it was sufficient to gently add a rinse after the $80 \%$ ethanol bead washes while the beads were on the magnet, incubate for 60 seconds, and remove the rinse before eluting the DNA off the magnet for 20 minutes at $37^{\circ} \mathrm{C}$. We integrated rinses into the protocol for Run $C$ in addition to doing two sequential 0.4x AMPure clean-ups before and two after end-repair (Supplementary Fig. 3) and using 4x the recommended amount of input DNA. Although Run B and Run C started with the same source of DNA, the amount of molecules $<10 \mathrm{~kb}$ in Run $\mathrm{C}$ was greatly depleted compared to Run B (Fig. 1D, Supplementary Fig. 5). Run C had proportionally fewer tiny events files than Run A (55.5\% of events files had fewer than 2000 events) that contained proportionally fewer events (1.24\%) than both Runs A and B (Table 1). There were proportionally $>2$-fold more base-called molecules $>50 \mathrm{~kb}$ and $>100 \mathrm{~kb}$ in Run C than in Run B, and Run C had the highest mean and median molecule sizes (Table 1, 
Supplementary Table 3$)$. The longest $2 \mathrm{D}$ read was $84.898 \mathrm{~kb}(\mathrm{Q}=8.87)$ and the longest $1 \mathrm{D}$ read was $139.864 \mathrm{~kb}(\mathrm{Q}=4.28)$. The base-called molecule $\mathrm{N} 50$ was $20.824 \mathrm{~kb}$ and there was a higher proportion $(25.9 \%)$ of base-called molecules $>10 \mathrm{~kb}$ than both previous runs, which made up $77.2 \%$ of the summed molecule length. The percent of base-called files with $2 \mathrm{D}$ reads $(28.2 \%)$ and the total output of the flow cell $(70.1 \mathrm{Mb}$ of summed molecule lengths) were both intermediary between Runs A and B, suggesting that there is a trade-off between read length and output/2D reads. However, it is possible that the differences in output and proportion of $2 \mathrm{D}$ reads amongst the runs were due to variation in library preparations and flow cell quality, though Runs B and C had a similar estimated number of active channels (Supplementary Table 3).

Each run produced event files (69 total, Supplementary Table 5) with too many events (>1 million) for base-calling and we investigated whether they were likely to represent megabase molecules. First we eliminated all multi-million event files that contained blocks of events repeated numerous times (Supplemental Fig. 6). This error is rare $(0-0.017 \%$ of all files; Supplementary Table 5$)$, but was more prominent in files with 100 thousand to 1 million events $(0-5.51 \%)$ than it was in files with $<100$ thousand events $(0 \%)$ and was most prominent in the files with $>1$ million events $(28.6-65.2 \%)$. Of the multi-million event files, 30 of the 69 did not contain this error (19 shown in Fig. 2A). Another concern is that large events files might arise from a faulty pore independent of a DNA molecule traversing the pore. To rule this out, we discarded 11 of the 30 remaining files that did not show evidence of a lead adapter profile (Fig. 2B). A third concern is that DNA molecules can become temporarily stuck in the pores leading to an accumulation of events from the same region of a DNA molecule. To determine how pervasive this issue might be in the remaining multi-million event files, we looked at how 
many times the base-caller decided two or more adjacent events corresponded to the same kmer (move=0, "stay") instead of advancing to a new kmer (move $\geq 1$ ) in files with $<1$ million events (Fig. 2C, Supplementary Fig. 7). In general, all base-called files with $\geq 531,779$ events had an average of $>80 \%$ " 0 moves" in the template and complement (Fig. 2C). Indeed, the base-called sequences that have $>500$ thousand events appear to have come from 12.687-196.362kb molecules, also reflected in their high event:nt ratios (2-76) (Supplementary Fig. 7). Thus, it is probable that the remaining 19 files that contained 1.1-5.2 million events correspond to molecules that were stuck.

Finally, we sought to understand how DNA might get stuck in pores. We hypothesized one possibility is that a highly stable DNA secondary structure known as a G-quadruplex $(\mathrm{G} 4)^{21}$ may form and block further translocation until it unfolds (Fig. 2D, Supplementary Fig. 8), resulting in an accumulation of measurements of a 5mer or set of 5 mers slightly upstream of the G4. Indeed, there is a significantly higher number of " 0 moves" near G4 motifs than near randomly selected locations even when controlling for read-specific effects (Supplementary Table 6A). Moreover, there is a significantly higher number of "0 moves" near G4 motifs on complement strands than near G4 motifs on template strands consistent with the higher propensity of G4-folding in single-stranded DNA $^{21}$ (Supplementary Table 6B). With each additional poly-G tract inside a G4 motif there are additional ways a G4 can form, increasing the probability that one will. Thus, if G4 structures are associated with DNA stalling, one might expect more "0 moves" near G4 motifs with more poly-G tracts. Indeed, there is also a significantly higher number of "0 moves" near G4 motifs that have $>4$ poly-G tracts than G4 motifs with only 4 G-tracts (Supplementary Table 6C). Finally, in an aggregate analysis looking at all template and complement reads, there is a clear enrichment of "0 moves" near G4 motifs with the 
highest enrichment (Run A) and shoulders (Runs B, C) slightly upstream (-9 to -27 nt) of the G4 motif (position 0) as expected (Fig. 2D, Supplementary Fig. 8). Nonetheless, it is clear that G4s are not the only way DNA molecules get stuck since there are many "0 moves" far away from and on different molecules than G4 motifs. Fortunately, since the base-caller can identify "0 moves", it is able to deal with DNA stalling, though there is a slight decrease in $Q$ with each increase in the proportion of called events that have " 0 moves" (Supplementary Fig. 7). Consistently, reads with G4 motifs appear to have a slightly lower average $Q$ than all reads (Supplementary Table 6D). Nonetheless, in future studies, "0 moves" might serve as an indicator of whether and how often all possible G4 motifs in a genome form in vitro in a single MinION experiment.

In conclusion, our data demonstrate that with our modified protocols the MinION can sequence as many ultra-long DNA molecules $>100 \mathrm{~kb}$ that make it intact to the nanopores. Importantly, we demonstrate that it is possible to obtain high quality 2D reads $>100 \mathrm{~kb}$ (e.g. 102.935kb, Q=8.74). Indeed, using our modified protocols, ONT internally obtained a $192 \mathrm{~kb}$ high quality $2 \mathrm{D}$ E. coli read that mapped to the reference genome. The modified protocols presented here will help others obtain similar read size distributions. 


\section{ACKNOWLEDGMENTS}

We thank Oxford Nanopore Technologies for including us in MAP, the MAP community for online discussions, Ben Raphael for providing computing resources for the MinION, and Mark Howison for helpful discussions. We gratefully acknowledge support from predoctoral fellowships from NSF GRFP (DGE-1058262) and NSF EPSCoR (grant\# 1004057) to J.M.U.

\section{AUTHOR CONTRIBUTIONS}

J.M.U. conceived, designed, and carried out the experiments, analyzed the data, wrote and utilized the associated "poreminion" software (https://github.com/JohnUrban/poreminion), and prepared the manuscript. J.B. performed and supplied mass-matings of flies for embryo collection and contributed to discussions about the project. C.E.L. provided guidance in statistics and experimental design. S.A.G. provided guidance in molecular techniques and experimental design, and helped prepare the manuscript. All authors read and approved the manuscript.

\section{COMPETING FINANCIAL INTERESTS}

JMU and SAG are members of the MinION Access Programme (MAP) and have received free reagents for nanopore sequencing. 


\section{REFERENCES}

1. Check Hayden, E. Pint-sized DNA sequencer impresses first users. Nature 521, 15-16 (2015).

2. Kilianski, A. et al. Bacterial and viral identification and differentiation by amplicon sequencing on the MinION nanopore sequencer. Gigascience 4, 12 (2015).

3. Loman, N. J. \& Watson, M. Successful test launch for nanopore sequencing. Nat. Methods 12, 303-304 (2015).

4. Quick, J. et al. Rapid draft sequencing and real-time nanopore sequencing in a hospital outbreak of Salmonella. Genome Biol. 16, 114 (2015).

5. Mikheyev, A. S. \& Tin, M. M. Y. A first look at the Oxford Nanopore MinION sequencer. Mol. Ecol. Resour. 14, 1097-1102 (2014).

6. Quick, J., Quinlan, A. R. \& Loman, N. J. A reference bacterial genome dataset generated on the MinION(TM) portable single-molecule nanopore sequencer. Gigascience 3, 22 (2014).

7. Goodwin, S. et al. Oxford Nanopore Sequencing and de novo Assembly of a Eukaryotic Genome. bioRxiv (Cold Spring Harbor Labs Journals, 2015).

doi:10.1101/013490

8. Ashton, P. M. et al. MinION nanopore sequencing identifies the position and structure of a bacterial antibiotic resistance island. Nat. Biotechnol. 33, 296-300 (2014).

9. Jain, M. et al. Improved data analysis for the MinION nanopore sequencer. Nat. Methods 12, 351-356 (2015).

10. Ammar, R., Paton, T. A., Torti, D., Shlien, A. \& Bader, G. D. Long read nanopore sequencing for detection of HLA and CYP2D6 variants and haplotypes.

F1000Research 4, 17 (2015).

11. Madoui, M.-A. et al. Genome assembly using Nanopore-guided long and errorfree DNA reads. BMC Genomics 16, 327 (2015).

12. Warren, R. L., Vandervalk, B. P., Jones, S. J. \& Birol, I. LINKS: Scaffolding genome assemblies with kilobase-long nanopore reads. bioRxiv (Cold Spring Harbor Labs Journals, 2015). doi:10.1101/016519

13. Bolisetty, M., Rajadinakaran, G. \& Graveley, B. Determining Exon Connectivity in Complex mRNAs by Nanopore Sequencing. bioRxiv (Cold Spring Harbor Labs Journals, 2015). doi:10.1101/019752 
14. Chiu, C. Y. et al. Rapid metagenomic identification of viral pathogens in clinical samples by real-time nanopore sequencing analysis. bioRxiv (Cold Spring Harbor Labs Journals, 2015). doi:10.1101/020420

15. Cao, M. D. et al. Real-time strain typing and analysis of antibiotic resistance potential using Nanopore MinION sequencing. bioRxiv (Cold Spring Harbor Labs Journals, 2015). doi:10.1101/019356

16. Szalay, T. \& Golovchenko, J. A. A de novo DNA Sequencing and Variant Calling Algorithm for Nanopores. bioRxiv (Cold Spring Harbor Labs Journals, 2015). doi:10.1101/019448

17. Sovic, I. et al. Fast and sensitive mapping of error-prone nanopore sequencing reads with GraphMap. bioRxiv (Cold Spring Harbor Labs Journals, 2015). doi:10.1101/020719

18. Loman, N. J., Quick, J. \& Simpson, J. T. A complete bacterial genome assembled de novo using only nanopore sequencing data. bioRxiv (Cold Spring Harbor Labs Journals, 2015). doi:10.1101/015552

19. Check Hayden, E. Nanopore genome sequencer makes its debut. Nature (2012). doi:10.1038/nature.2012.10051

20. Check Hayden, E. Data from pocket-sized genome sequencer unveiled. Nature (2014). doi:10.1038/nature.2014.14724

21. Huppert, J. L. Structure, location and interactions of G-quadruplexes. FEBS J. 277, 3452-8 (2010). 


\section{Figures:}

\section{Sequencing ultra-long DNA molecules with the Oxford Nanopore MinION}

John M. Urban, Jacob Bliss, Charles E. Lawrence, Susan A. Gerbi

\section{Figure 1:}
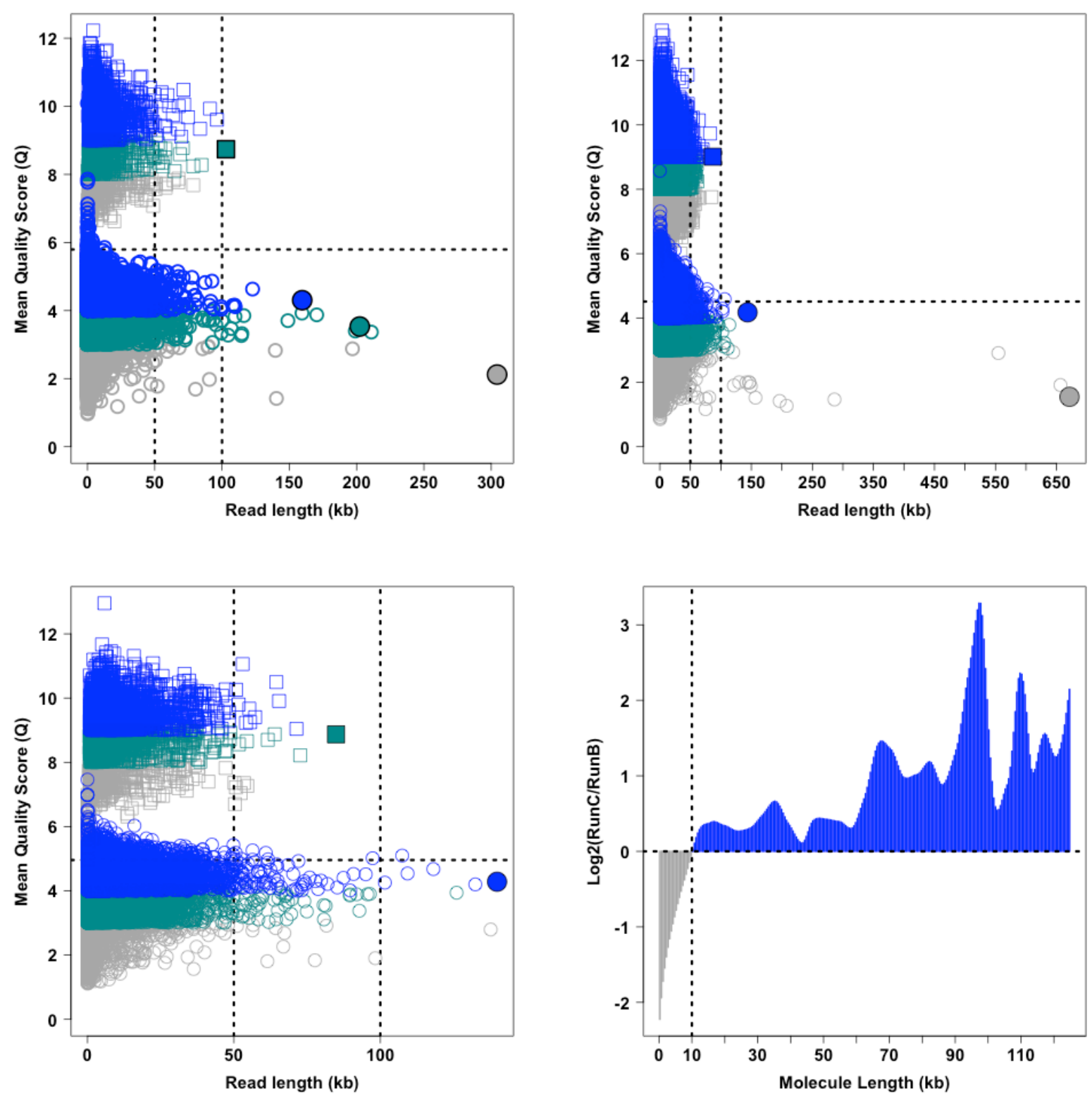

Figure 1: Read lengths and mean quality scores $(Q)$ across runs

Read length vs. Mean Quality Score (Q) for (A) Run A, (B) Run B, and (C) Run C. In each, the distributions for both $2 \mathrm{D}$ reads (squares; concentrated above $\mathrm{Q}=6$ ) and $1 \mathrm{D}$ reads (circles; concentrated below $Q=6$ ) are shown. For $2 D$ reads, blue is used for reads with $Q>9$, cyan for $Q$ between 8 and 9 , and grey for $Q<8$. For $1 D$ reads, blue is used for $Q \geq 4$, cyan for $Q$ between 3 and 4 , and grey for $Q<3$. Filled-in squares and circles indicate reads highlighted 
in the main paper for their length and Q. The horizontal dashed line marks the minimum 2D Q. The vertical dashed lines denote $50 \mathrm{~kb}$ and $100 \mathrm{~kb}$. (D) The log2 fold change of Run $\mathrm{C}$ over Run B of the proportion of total summed molecule length plotted as a function of molecule length. Run B and Run C used the same source of DNA, but differed in library preparation. Run $\mathrm{C}$ used a new rinse step during all AMPure clean-ups as well as took additional advantage of sequential AMPure rounds in each clean-up step. The result was depleting molecules $<10 \mathrm{~kb}$, thereby enriching longer molecules proportionally. 
Figure 2:

A

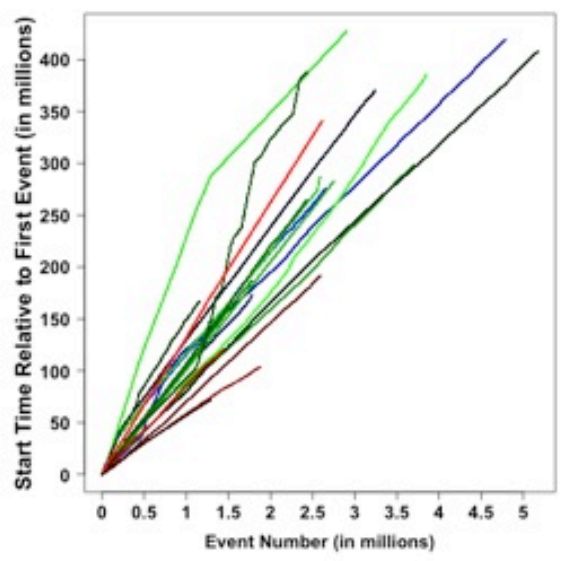

C

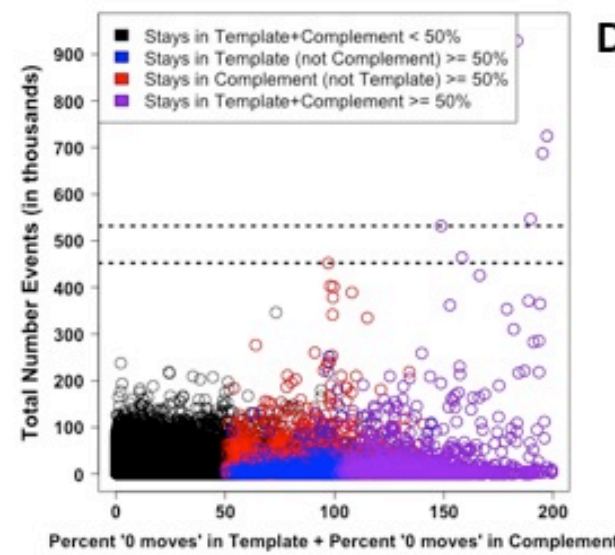

B
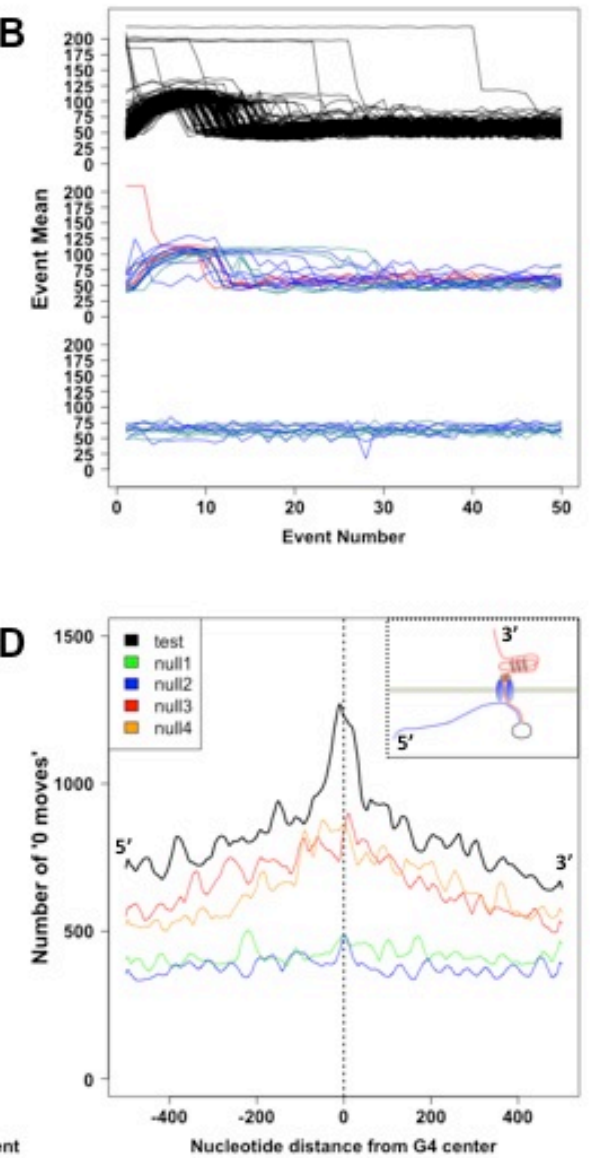

Figure 2: Dissecting files with >1 million events and a role of G-quadruplexes in DNA stalling.

(A) Multi-million event files were first filtered to remove files with artificially high event counts due to repeated blocks of events. Shown are the start times as a function of event number for the multi-million event files that did not have repeated blocks of events and that had evidence of the lead adapter (i.e. middle row of 2B). Different colors represent different molecules. Shades of blue are from Run A, shades of green are from Run B, and shades of red are from Run C. (B) The event means of the first 50 events for: (Top) 150 randomly sampled (50 from each run), high quality ( $Q \geq 9)$ 2D reads; (Middle) multi-million event files that did not have repeated events and do show evidence of the lead adapter; (Bottom) multi-million event files that did not have repeated events and do NOT show evidence of the lead adapter. Red lines are from Run A, blue lines are from Run B, and cyan lines are from Run C. (C) The total number of events plotted as a function of the summed percentages of base-called events in the template and complement ( $\mathrm{T}$ and $\mathrm{C}$ ) reads that had "0 moves". Notably all files with $\geq 452301$ events (bottom dashed line) had on average $\geq 50 \%$ base-called events with " 0 moves" in the $\mathrm{T}$ and $\mathrm{C}$ reads and all files with $\geq 531779$ events (top dashed line) had on average $\geq 80 \%$ base-called events with " 0 moves" in the $T$ and $\mathrm{C}$ reads. (D) The number of " 0 moves" as a function of proximity to G4 motif centers (test condition) or the centers of randomly selected positions (null 1-4 conditions). Briefly, positions were randomly selected on any template or complement ( $\mathrm{T}$ or $\mathrm{C}$ ) read without a G4 motif ('null 1'), on any T or C read including those with G4 motifs ('null 2'), on the same read the G4 motif originated ('null 3'), and on the same read the G4 motif originated ensuring it did not overlap the G4 motif ('null 4'). The inset at the top right depicts the 
orientation $\left(5^{\prime}-3^{\prime}\right)$ of a DNA molecule going through a pore from the top chamber to the bottom chamber and how a G4 might cause a DNA molecule to stall. 
Table 1: This table summarizes statistics and values discussed in the main text.

\begin{tabular}{|c|c|c|c|}
\hline & Run A & Run B & Run C \\
\hline Number Events Files & 27667 & 82040 & 12536 \\
\hline Number Base-Called (\% of events files) & $9935(35.9 \%)$ & $64282(78.35 \%)$ & $8941(71.3 \%)$ \\
\hline Number of molecules $>50 \mathrm{~kb}$ & 139 & 396 & 119 \\
\hline Number of molecules $>100 \mathrm{~kb}$ & 21 & 24 & 8 \\
\hline Longest $2 \mathrm{D}$ read (Q) & $\begin{array}{l}102.935 \mathrm{~kb} \\
(8.74)\end{array}$ & $\begin{array}{l}86.797 \mathrm{~kb} \\
(9.01)\end{array}$ & $\begin{array}{l}84.898 \mathrm{~kb} \\
(8.87)\end{array}$ \\
\hline Longest $2 D$ read $(Q>9)$ & $\begin{array}{l}96.237 \mathrm{~kb} \\
(9.6)\end{array}$ & $\begin{array}{l}86.797 \mathrm{~kb} \\
(9.01)\end{array}$ & $\begin{array}{l}71.830 \mathrm{~kb} \\
(9.04)\end{array}$ \\
\hline Longest $1 D$ read (Q) & $\begin{array}{c}304.309 \mathrm{~kb} \\
(2.12)\end{array}$ & $\begin{array}{c}671.219 \mathrm{~kb} \\
(1.55)\end{array}$ & $\begin{array}{c}139.864 \mathrm{~kb} \\
(4.28)\end{array}$ \\
\hline Longest $1 \mathrm{D}$ read $(Q>3.5)$ & $\begin{array}{l}202.293 \mathrm{~kb} \\
(3.53)\end{array}$ & $\begin{array}{l}143.763 \mathrm{~kb} \\
(4.17)\end{array}$ & $\begin{array}{l}139.864 \mathrm{~kb} \\
(4.28)\end{array}$ \\
\hline Total summed molecule length & $49.8 \mathrm{Mb}$ & $386.9 \mathrm{Mb}$ & $70.1 \mathrm{Mb}$ \\
\hline Molecule N50 & $25.238 \mathrm{~kb}$ & $13.553 \mathrm{~kb}$ & $20.824 \mathrm{~kb}$ \\
\hline Percent of base-called molecules $>10 \mathrm{~kb}$ & $14.4 \%$ & $15.8 \%$ & $25.9 \%$ \\
\hline $\begin{array}{l}\text { Percent of summed molecule lengths } \\
\text { from base-called molecules }>10 \mathrm{~kb}\end{array}$ & $79.2 \%$ & $58.5 \%$ & $77.2 \%$ \\
\hline Percent of molecules with 2D reads & $21.9 \%$ & $49.8 \%$ & $28.2 \%$ \\
\hline Percent of events files with $<2000$ events & $87.2 \%$ & $40.2 \%$ & $55.5 \%$ \\
\hline $\begin{array}{l}\text { Percent of total summed events in events } \\
\text { files with }<2000 \text { events }\end{array}$ & $3.41 \%$ & $2.48 \%$ & $1.24 \%$ \\
\hline
\end{tabular}




\section{Supplementary Materials:}

\section{Sequencing ultra-long DNA molecules with the Oxford Nanopore MinION}

John M. Urban, Jacob Bliss, Charles E. Lawrence, Susan A. Gerbi

\section{Table of Contents:}

Supplementary Methods

Supplementary Figures 


\section{Supplementary Methods}

Overview of genomic DNA isolation, MinION sequencing, and analysis:

We have been developing our protocols and reference-free analyses while collecting MinION data for a future assembly of the fungus gnat (Sciara coprophila) genome. All libraries were prepared with the SQK-MAP004 kit and reads were base-called with Metrichor 1.12 r7.X 2D-basecalling XL. In our analyses, we characterize the full distribution of reads as well as the pre-base-called events files. Reads were filtered to remove those with errors in event timing (repeated blocks of events) and those that were not base-called due to having too few events $(<200)$, too many events ( $>1$ million), or "no template" (Supplementary Table 5). The rare error that leads to blocks of events being repeated numerous times is easily identified by events with earlier start times than preceding events and therefore we often refer to it as the "time error". It is important to point out that the software issue that led to this error in a small number of files has since been resolved and will not occur in future experiments. Filtering and data analyses were carried out using our open source MinION toolset called "poreminion" (https://github.com/JohnUrban/poreminion) as well as in $\mathrm{R}^{1}$ and using poretools ${ }^{2}$.

\section{DNA Extraction:}

Genomic DNA (gDNA) was extracted from $51 \mathrm{mg}$ (sample 1, Run A) and $53 \mathrm{mg}$ (sample 2 , Runs $B$ and $C$ ) of synchronized 2 day old Sciara male embryos from $\sim 30$ mass matings each that were extensively washed with sterile TE, pH 8.5. There were 5-8 adult males and 10 male-producing adult females per mass mating (note that Sciara females have either only male or only female offspring, which we can control). The washed male embryos were homogenized with a blue pestle inside a $1.5 \mathrm{ml}$ microfuge tube in $200 \mu \mathrm{l}$ DNAzol (Life Technologies): 5-10 gentle strokes for sample 1; 20 strokes for sample 2. Then $800 \mu \mathrm{l}$ more DNAzol was added to the tube for $1 \mathrm{ml}$ DNAzol total. The homogenate from sample 2 (not sample 1) was vortexed (full speed, 30 seconds) to further facilitate homogenization and lysis. The DNAzol homogenate was (for both samples 1 and 2) incubated with $5 \mu \mathrm{l}$ RNase A Solution (Qiagen) for 10 minutes at $37^{\circ} \mathrm{C}$, followed by $5 \mu \mathrm{l}$ Puregene Proteinase K (Qiagen) for 10 minutes at $37^{\circ} \mathrm{C}$. The DNAzol homogenate was then centrifuged at $10,000 \mathrm{xg}$ for 10 minutes to pellet debris. The supernatant was transferred to a new $1.5 \mathrm{ml}$ tube [using a wide-bored tip (Axygen: Max Recovery, DNase/RNase-free, Sterile, Wide-bore tips) for sample 1 and regular tip (Olympus: Low-binding, RNase/DNase-free, Sterile, Barrier tips) for sample 2] and $500 \mu \mathrm{l} 100 \%$ ethanol was added. The tube was capped and slowly inverted 50 times, incubated at room temperature for 2 minutes, then on ice for 2 minutes. Precipitated DNA was pelleted at $18,000 \times$ for 10 minutes. The DNA pellet was washed twice with $80 \%$ ethanol, air-dried for 30 seconds, and re-suspended in 1xTE, pH 8. Sample 2 (not sample 1) was vortexed (full speed, 30 seconds) to help facilitate re-suspension. Furthermore, to help re-suspension of high molecular weight DNA, both samples were incubated at $37^{\circ} \mathrm{C}$ for 1 hour before an overnight incubation at room temperature and storage at $4^{\circ} \mathrm{C}$ (in $1 \mathrm{XTE}, \mathrm{pH} 8$ ) for up to 2 weeks before use.

\section{AMPure beads clean-up \#1:}

After DNA extraction, but before beginning the MinION library preparation, an appropriate volume of the DNAzol-extracted DNA was used to obtain 3, 3.6, and $4 \mu \mathrm{g}$ of gDNA for Runs $A, B$, and $C$ respectively. For all runs $(A, B$, and $C)$, the DNA was cleaned with 1.0x AMPure beads (Beckman Coulter Agencourt) with the following specifications. An equal volume (1.0x) of AMPure beads was added to DNA in 1X TE 
( $\mathrm{pH} 8)$, incubated for 15 minutes at room temperature (RT), then pelleted on a magnetic rack for 5 minutes (RT) before removing the supernatant. The beads were then washed with $500 \mu \mathrm{l}$ buffered $80 \%$ ethanol (always buffered with $10 \mathrm{mM}$ Tris- $\mathrm{Cl}, \mathrm{pH}$ 8) followed by a second wash with $200 \mu \mathrm{l}$ buffered $80 \%$ ethanol, a brief gentle spin in a microfuge to collect any remaining $80 \%$ ethanol at the bottom of the tube, 1 minute re-pelleting on a magnet, removal of the remaining buffered $80 \%$ ethanol collected at the bottom of the tube without disturbing beads, air drying 7 minutes, re-suspending in $175 \mu$ Ultra Pure Water (UPW, Life Technologies UltraPure DNase/RNase-Free Distilled Water) at $37^{\circ} \mathrm{C}$ for 20 minutes to help ensure long DNA elutes off the beads, pelleting the beads on a magnet for 5 minutes, then transferring $174 \mu$ l supernatant with DNA to a new tube (for PreCR). For Run A, wide-bored tips and gentle pipetting were used. For Runs B and C, normal tips and normal pipetting were used. For Run C, directly before eluting the DNA off the beads, a "rinse" of $200 \mu 10 \mathrm{mM}$ Tris $(\mathrm{pH} 8)$ was gently added to the tube wall opposite the magnetically pelleted beads, incubated at room temperature for 60 seconds, and gently removed. This is an additional rinse step used to help deplete DNA $<10 \mathrm{~kb}$ (see Supplementary Fig. 4B, Fig. 1D, and Supplementary Fig. 5).

\section{PreCR DNA Repair:}

Since 3-4x the ONT-recommended amount of starting material was used, PreCR (New England BioLabs, NEB) was performed in double the volume, with double the reagents, for double the time (all relative to ONT protocol): $200 \mu$ l total volume with $174 \mu \mathrm{l}$ AMPure cleaned DNA, $20 \mu \mathrm{l}$ 10x Thermopol buffer, $2 \mu \mathrm{l} 100 x \mathrm{NAD}+, 2 \mu \mathrm{l} 10 \mathrm{mM}$ dNTPs, and $2 \mu$ PreCR Repair Mix. The reaction was incubated at $37^{\circ} \mathrm{C}$ for $\geq 60$ minutes.

\section{AMPure beads clean-up \#2:}

For Runs $A$ and $B$, we proceeded as in clean-up \#1, only with a 0.4x AMPure beads ratio and transferring $85 \mu \mathrm{l}$ to a new tube at the end (for End-Repair). For Run C, sequential $0.4 x$ clean-ups were performed in the following way. In the first $0.4 x$ AMPure clean-up, the beads were air-dried for only 2 minutes (after the buffered $80 \%$ ethanol washes) before adding $140 \mu \mathrm{l} 0.4 \mathrm{x}$ AMPure solution (100 $\mu \mathrm{l}$ UPW, $40 \mu \mathrm{l}$ AMPure beads), gently re-suspending the beads in the second $0.4 x$ solution, and proceeding as in clean-up \#1 for Run C. Importantly, a "rinse" was again performed before eluting DNA off the beads. Specifically, $100 \mu \mathrm{l} 10 \mathrm{mM}$ Tris- $\mathrm{Cl}(\mathrm{pH} 8)$ was added to the tube wall opposite the pelleted beads, and incubated for 30 seconds at room temperature while the tube remained on the magnet before gently removing the rinse. Again (for all runs), elution off the beads (into $85 \mu \mathrm{U}$ UPW) was performed for a longer time and a higher temperature than manufacturer recommendations to facilitate the elution of long DNA $\left(\geq 20\right.$ minutes, $\left.37^{\circ} \mathrm{C}\right)$.

\section{End-Repair:}

The NEBNext End-Repair Module (NEB) was used: $85 \mu \mathrm{l}$ of DNA from the previous AMPure step, $10 \mu \mathrm{l}$ NEBNext End-Repair Reaction Buffer (10X), and $5 \mu \mathrm{l}$ NEBNext End-Repair Enzyme Mix. The reaction was incubated for 30 minutes at $22^{\circ} \mathrm{C}$.

\section{AMPure beads clean-up \#3:}

For Run A, we proceeded as in \#2, except that elution was in $30 \mu \mathrm{l}$. For Run B, two sequential 0.4x AMPure clean-ups were performed as done for Run $\mathrm{C}$ in \#2 (but with no rinse). For Run $\mathrm{C}$, we proceeded as Run $\mathrm{C}$ in \#2 with 2 sequential $0.4 x$ washes and the rinse step. Specifically, $100 \mu 10 \mathrm{mM}$ Tris- $\mathrm{Cl}(\mathrm{pH} 8)$ was added to the tube wall opposite 
the pelleted beads, then incubated for 30 seconds at room temperature while the tube remained on the magnet before gently removing the rinse and eluting for $\geq 20$ minutes at $37^{\circ} \mathrm{C}$. For all runs, DNA was eluted into $30 \mu \mathrm{l} 10 \mathrm{mM}$ Tris- $\mathrm{Cl}(\mathrm{pH} 8)$.

\section{dA-tailing:}

The NEBNext dA-Tailing Module (NEB) was used: $25 \mu$ I DNA from the previous AMPure step, $3 \mu \mathrm{l}$ NEBNext dA-Tailing Reaction Buffer (10X), and $2 \mu$ Klenow Fragment $\left(3^{\prime} \rightarrow\right.$ $5^{\prime}$ exo-). The reaction was incubated for 30 minutes at $37^{\circ} \mathrm{C}$.

\section{Adapter Ligation:}

The following were combined: $30 \mu \mathrm{d}$ dA-tail reaction, $8 \mu \mathrm{l}$ UPW, $10 \mu \mathrm{I}$ ONT SQKMAP004 Adapter Mix, $2 \mu$ ONT SQK-MAP004 HP adapter, $50 \mu \mathrm{l}$ 2X Blunt/TA Ligase Master Mix. The reaction was incubated for 30 minutes at $22^{\circ} \mathrm{C}$.

\section{Enrichment of HP-ligated DNA with His-Beads:}

$550 \mu \mathrm{L}$ UPW was added to $550 \mu \mathrm{L}$ SQK-MAP004 2X Wash Buffer (this is called " $1 \mathrm{X}$ Wash Buffer"), then mixed by inverting 10 times, and briefly spun down in a microfuge. "His-beads" (Dynabeads His-tag Isolation and Pulldown; Life Technologies) were resuspended by vortexing for 30 seconds. Then $10 \mu \mathrm{l}$ of re-suspended beads was transferred to a $1.5 \mathrm{ml}$ Protein LoBind tube (Eppendorf), and combined with $250 \mu \mathrm{l} 1 \mathrm{X}$ Wash Buffer. The tube was placed on a magnet for 2 minutes before aspirating off the supernatant. The his-beads were re-suspended in another $250 \mu \mathrm{l} 1 \mathrm{X}$ Wash Buffer and placed on the magnet for another 2 minutes before removing the supernatant. The twice-washed and pelleted his-beads were re-suspended in $100 \mu$ undiluted (2X) Wash Buffer and are referred to as the "washed his-beads". $100 \mu \mathrm{l}$ "washed his-beads" was added to the $100 \mu$ ladapter ligation reaction, mixed by gentle pipetting (wide-bore tips), and incubated at $22^{\circ} \mathrm{C}$ for 5 minutes. The his-beads were then pelleted using a magnetic rack for 2 minutes before removing the supernatant, then washed twice with $250 \mu \mathrm{l}$ 1X Wash Buffer (with 30 second incubations). The tube was briefly spun in a microfuge to collect excess Wash Buffer at the bottom of the tube, which was then placed in the magnetic rack for 2 minutes before removing the excess buffer. The pelleted his-beads were re-suspended in $30 \mu \mathrm{l}$ of Elution Buffer with gentle pipetting using a wide-bored tip, adding the buffer close to the his-beads (to avoid any residual wash buffer on the sides of the tube). The elution was incubated for 10 minutes at $22^{\circ} \mathrm{C}$ before pelleting with a magnetic rack for 2 minutes. The eluted supernatant (called the "Pre-Sequencing Mix" (PSM)) was transferred to a new Protein LoBind tube.

\section{Pre-sequencing Mix (PSM):}

Before the PreCR step, Run A started with $3000 \mathrm{ng}$ gDNA, Run B started with $3600 \mathrm{ng}$, and Run C started with $4080 \mathrm{ng}$. All had A[260/280] and A[260/230] >1.8 prior to PreCR and $>2.0$ after subsequent clean-up steps during the library preparation as determined by Nanodrop (Thermo Scientific). Each sample preparation ended with between 300400 ng DNA in the PSM as measured by Qubit dsDNA HS (Life Technologies).

\section{Loading the Sequencing Mix (SM):}

Sequencing Mix (SM) was made using 3-6 $\mu \mathrm{l} \mathrm{PSM,} \mathrm{3-4} \mu \mathrm{l}$ Fuel, and EP buffer up to 150 $\mu \mathrm{l}$. SM was made fresh for loading/re-loading at various intervals 6-7 times throughout each run. See Supplementary Table 4B for exact details. 


\section{Base-calling:}

MinION events data for each DNA molecule is stored in an individual "fast5" file. All fast5 files were base-called using the Metrichor 1.12 r7.X 2D-basecalling XL protocol. Metrichor returns fast5 files that are updated with base-calling information.

\section{Filtering base-called fast 5 files:}

Metrichor (ONT base-caller) returns updated fast5 files into two folders: "pass" and "fail". "Pass" contains only fast 5 files where $2 \mathrm{D}$ base-calling was successful and the mean quality of the $2 \mathrm{D}$ read is $>9$. Everything else (including other fast5s containing $2 \mathrm{D}$ reads with $Q<9$, fast5s with only $1 \mathrm{D}$ base-calling, and fast5s that failed base-calling) goes into the "fail" folder. To analyze all base-called molecules, we filtered the contents of the fail folder to remove un-basecalled files and further filtered to remove any base-called files that contained the "time error" where a block of events is repeated. Both were accomplished by using our toolset for working with MinION data, called "poreminion" (which, for some of its functionality, sources the Fast5File classes from poretools ${ }^{2}$ ):

\$ poreminion uncalled -m -o fail-filter fail/

$\$$ poreminion timetest $-\mathrm{m}-\mathrm{o}$ fail-filter fail/

The syntax for both subcommands is:

poreminion subcommand options (-m -o outprefix) target-directory (to search).

The "uncalled" subcommand identifies all fast5s that were not base-called due to either: (1) too many events, (2) too few events, or (3) no template found. The flag "-o" gives a prefix to poreminion, which writes text files containing the names of the fast5s in each of the above categories in addition to a summary statistics file describing how many fast5s were searched, how many were assigned to each category, as well as the minimum, maximum, median, and mean number of events found in files of each category. It also reports the number of events for all files with too many events to base-call. The "-m" flag tells poreminion to not only report the names of the un-basecalled files, but to also move them into their own folders. The "timetest" subcommand searches for files with repeated blocks of events, which are identified by looking at the start times of all events in a fast 5 file. If a fast 5 file contains an event start time that is earlier than the event that preceded it, then the fast 5 file contains this error and is reported ("-o") and moved to a folder for files with this error ("-m").

Obtaining molecule size, mean quality score (Q), other statistics, and plotting:

Each base-called fast5 file from a MinION run describes data from a single molecule, yet there can be up to 3 reads per file: template, complement, and 2D. We define the molecule size as the length of the $2 \mathrm{D}$ read if present, the length of the template read if there is only a template read present, and the length of longer of template and complement reads when both are present in the absence of a $2 \mathrm{D}$ read. Thus, the only time there is a choice is in the latter situation. The majority $(68-86 \%$, Supplementary Table 2, Supplementary Fig. 1) of files with a template and complement sequence have a 2D read, all of which have a template:complement sequence length ratio between 0.5 and 2. Moreover, most files that contain both template and complement sequences with a sequence length ratio between 0.5 and 2 have 2D reads (Supplementary Fig. 1). This means that when a choice needs to be made between the template and complement they are vastly different sizes. The complement can be much smaller than the template, for example, when there is a nick in the complement strand. The template can be much 
shorter than the complement, for example, when the motor protein on the Y-adapter falls off allowing the template to zip through until it is caught by the hairpin motor protein. In these situations, the longer read better represents the size of the molecule that was sequenced. Importantly, molecule size allows for a single/non-redundant length from each base-called fast5 file (i.e. single molecule) to compute statistics on, such as the summed length of sequenced molecules and molecule N50, in addition to statistics computed on all read types. This molecule size estimate as well as many descriptive metrics of the fast5 file were obtained with the poreminion subcommand "fragstats" after combining all base-called files from the pass and fail folders.

\$ poreminion fragstats all-basecalled-files/ > fragstats.txt

The "fragstats" subcommand gives a tab-delimited output where each line describes an individual fast5 file (or single molecule) with the following columns (for version 0.4.3).

$1=$ read name

2 = estimated molecule/fragment size

3 = number input events

$4=$ if complement detected

$5=$ if $2 \mathrm{D}$ detected

$6=$ number of template events

$7=$ number of complement events

$8=$ length of $2 \mathrm{D}$ sequence

$9=$ length of template sequence

$10=$ length of complement sequence

$11=$ mean quality score of $2 \mathrm{D}$ sequence

$12=$ mean quality score of template sequence

$13=$ mean quality score of complement

$14=$ ratio of number template events to number complement events

$15=$ channel number molecule traversed

$16=$ heat sink temperature while molecule traversed

$17=$ number of called template events (after events pruned during base-calling)

18 = number of called complement events (after events pruned during base-calling)

$19=$ number of skips in template (number 0 )

20 = number of skips in complement (number 0 moves)

$21=$ number of stays in template

22 = number of stays in complement

$23=$ strand score template

$24=$ strand score complement

$25=$ number of stutters in template

$26=$ number of stutters in complement

The tab-delimited fragstats.txt file was then brought into $\mathrm{R}$ to make most plots (Fig. 1, Supplementary Figures 1,2, 3, and 7). The fragstats file was also summarized (generating many of the statistics reported such as in Table 1 and Supplementary Tables 1A-C, 2, 3A-E) using:

\$ poreminion fragsummary -f fragstats.txt 


\section{Analyzing files with too many events (>1 million) to base-call:}

Time Error:

Poreminion was used to extract the event start times from the multi-million event fast5 files into text files (columns of poreminion ouput = event mean, event standard deviation, event start time, event duration), which were brought into $\mathrm{R}^{1}$ for visualization.

$\$$ poreminion events $-\mathrm{f} 5$ target.fast5 | cut $-\mathrm{f} 3>$ start.times.txt

In the directory with the fast5 files that had too many events to base-call (this directory was made by "uncalled" filtering above), the poreminion "timetest" subcommand was used to further filter these multi-million event fast5s to keep only those without the "time error'. Time errors (and lack thereof) were visualized in $R$ from events text files extracted with the above poreminion command.

\section{Lead adapter:}

The first 50 events of the remaining multi-million event fast 5 files were obtained with poreminion (poreminion events $-f 5$ target.f5) and searched for evidence of the lead adapter event mean profile by comparing to 150 randomly sampled (50 from each run $A, B, C)$ pass fast5s (containing high quality 2D reads). Since there were so few multimillion event files, it was sufficient to manually separate ones with the lead adapter profile from ones that did not. However, we also found that the simple rule of requiring that there be 2 or more events within the first 15 events that have means >80 was sufficient to automatically separate the files this way for visualization.

\section{Looking at the number of "0 moves" (stays) vs. length and/or quality:}

The fragstats.txt file produced above was brought into $R^{1}$ for the various plots comparing the number of stays ("0 moves") with other features such as read length, number of events, number of called events, and mean quality scores (Q).

\section{Identifying G4 motif positions in template and complement reads:}

The poreminion subcommand " $\mathrm{g} 4$ " was used in the following way:

\$ poreminion g4 -minG 3 -maxN 7 --numtracts --noreverse -f5 all/ > g4s.bed

This subcommand uses the quadparser, ${ }^{3,4}$ regular expression, $\mathrm{G}_{3+-} \mathrm{N}_{1-7} \mathrm{G}_{3+} \mathrm{N}_{1-7} \mathrm{G}_{3+} \mathrm{N}_{1-}$ ${ }_{7} \mathrm{G}_{3+}$ (regular expression in Python: '([gG]\{3,\}lw\{1,7\})\{3,\}[gG]\{3,\}') to search the sequences inside fast5 files for $\mathrm{G} 4$ motifs $\left(\mathrm{G}_{3+}\right.$ is specified by "-minG 3 " and $\mathrm{N}_{1-7}$ is specified by "-maxN 7"). The "-noreverse" option specifies to only search the sequence given (not its complement), or in other words it specifies to NOT also search for the "C4" motif: $\mathrm{C}_{3+}-\mathrm{N}_{1-7} \mathrm{C}_{3+} \mathrm{N}_{1-7} \mathrm{C}_{3+} \mathrm{N}_{1-7} \mathrm{C}_{3+}$. The "-numtracts" option reports the number of poly-G tracts inside a given $\mathrm{G} 4$ motif as the Python regular expression (above) searches for 4 or more adjacent poly-G tracts separated by 1-7 nucleotides. The more poly-G tracts, the more possible ways a G4 structure could form. For example, observing five poly-G tracts does not simply indicate two overlapping G4 motifs that can form only two G4 structures, but rather "5 choose 4" (5) ways to choose four of those five poly- $G$ tracts multiplied by the number of ways 4 poly- $G$ tracts can fold together into a G4 structure (e.g. there are parallel and anti-parallel arrangements) as well as the multiplicative possibilities when varying the number and position of consecutive Gs $(\geq 3)$ used in the chosen poly-G tracts. Keeping track of the number of poly-G tracts inside each G4 motif allowed us to separate the G4 motifs into two groups: 
those with 4 poly- $G$ tracts and those with $>4$ for the subsequent statistical analysis testing which group has more "0 moves" associated with it on average.

Identifying positions of stays (" 0 moves") in template and complement reads:

The poreminion subcommand "staypos" was used as follows:

\$ poreminion staypos all/ > stays.bed

This subcommand goes through the base-called events of template and complement strands while keeping track of the index of each event relative to the output sequence (by accounting for base-caller "moves" of 0-5) and reporting the positions in the sequence that correspond to " 0 moves" in the base-called events. Specifically, it reports the coordinates of the 5 mer corresponding to the " 0 move" in BED format (for example, if a 5 mer starts at position 0 , its end position is 5).

\section{Comparing G4 and Stay positions in template and complement reads:}

For plotting, windowBed from BEDtools ${ }^{5}$ was used to obtain all stay positions within 500 nucleotides of a G4 motif (done independently for each of the three runs):

\$ windowBed -a g4s.bed -b stays.bed -w $500>$ g4s.stays.500.windowbed

The resulting file contains lines with pairs of entries for the G4 motif position and stay ("0 move") position for each pair that is within 500 nucleotides of each other. This file was brought into $R^{1}$ where distances between $\mathrm{G} 4$ centers and 'stay' centers (i.e. the middle nucleotide of a 5mer) from G4-stay pairs were calculated as the distance between their centers. Centers were found by subtracting 1 from the end position of each BED entry (to account for BED format), then taking the mean of the start and resulting end positions. Histogram information was obtained by, for example, hist(distances, breaks $=\operatorname{seq}($ from $=-650.5$, to $=650.5$, by=1), which results in the histogram midpoints being integers from -650 to 650 . Histogram counts were lightly loess smoothed (loess(hist.counts hist.mids, span=0.05).

Four null distributions were considered -- for each G4 motif, a site of the same length was selected uniformly at random from: (null 1) any template or complement read with no G4 motifs, (null 2) any template or complement read, (null 3) anywhere within the same read the G4 motif was on, (null 4) anywhere within the same read the G4 motif was that did not overlap the G4 motif coordinates. These coordinates were selected with BEDtools ${ }^{5}$ :

\$ shuffleBed -noOverlapping -excl readswithg4.bed -i g4s.bed -g template-andcomplement-reads > g4s.shuffled.nonG4reads.bed

\$shuffleBed -noOverlapping -i g4s.bed -g template-and-complement-reads > g4s.shuffled.allreads.bed

\$shuffleBed -noOverlapping -chrom -i g4s.bed -g template-and-complement-reads > g4s.shuffled.sameread.bed

\$shuffleBed -allowBeyondChromEnd -noOverlapping -chrom -excl g4s.bed -i g4s.bed -g template-and-complement-reads > g4s.shuffled.sameread.notoverG4.bed 
windowBed from BEDtools was used as above to collect pairs of randomly selected locations and "0 moves" that were within 500 nucleotides of each other. Histogram counts and smoothing was same as above. These null distributions were plotted on same plots as above.

For statistical analyses, we compared the number of "0 moves" within 50 nucleotides of the G4 motifs with their matched null positions from the four different null distributions. These counts were obtained using windowBed from BEDtools ${ }^{5}$. For example:

windowBed -c -a g4s.bed -b stays.bed -I 50 -r $50>$ g4s.stays.50.counts.txt

windowBed -c -a nulls.bed -b stays.bed -I 50 -r $50>$ nulls.stays.50.counts.txt

For each of the four nulls described above, the pairs of counts from G4 motifs and the null were used as input to the "sign test" as well as the Wilcoxon signed rank test in $\mathrm{R}^{1}$. Note that there seems to be more "0 moves" on reads with G4 motifs in general. The fourth null (null 4: selecting a random position on the same read as the G4 motif that does not overlap the G4 motif) serves as the best matched pairs, controlling for any read-specific effects and still all p-values were significant (Supplementary Table 6A). To test the hypothesis that G4 motifs on the complement strand associated with more "0 moves" than G4 motifs on the template strand, the counts for each of these group was used as input to the Wilcoxon rank sum test in $\mathrm{R}^{1}$ (Supplementary Table 6B). To test the hypothesis that $\mathrm{G} 4$ motifs with $>4$ poly- $G$ tracts were associated with higher " 0 move" counts than G4 motifs with only 4 poly-G tracts, the counts for each of these groups was used as input to the Wilcoxon rank sum test in $\mathrm{R}^{1}$ (Supplementary Table 6C). 


\section{Supplementary Figures}

\section{Supplementary Figure 1}
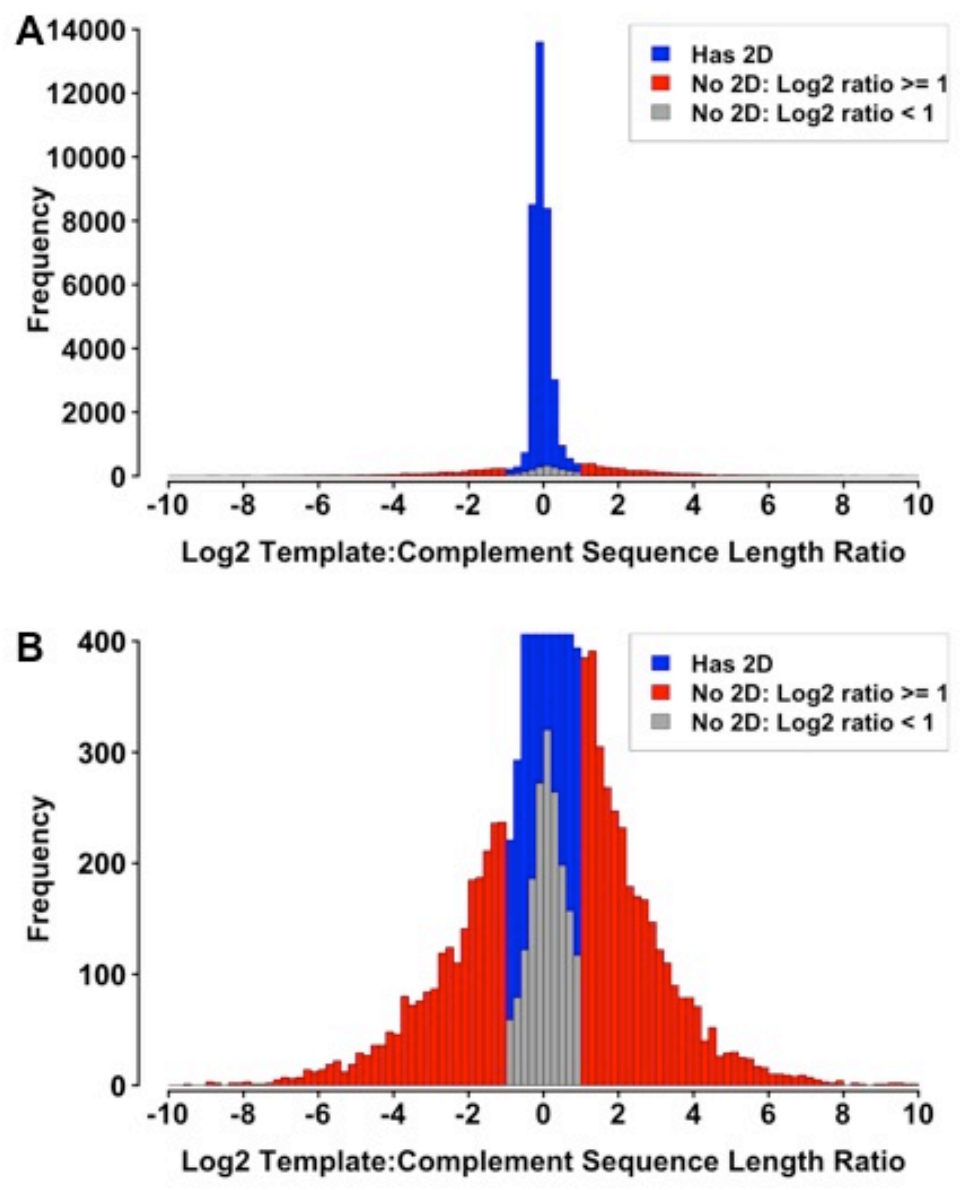

Supplementary Figure 1: Distribution of Log2(template:complement) for basecalled fast 5 files that contain both template and complement reads.

(A) shows a zoomed-out view of a histogram of Log2(ratio of template read length to complement read length) for all base-called fast 5 files that have both template and complement reads. The histogram shows the number (frequency) of fast5 files as a function of Log2(ratio). (B) shows a zoomed-in view of the bottom of the same histogram as in A. Most fast5 files that have both template and complement reads also have $2 \mathrm{D}$ reads (blue). For those that do not, most have template-to-complement read length ratios that are either too big $(>2$, or $>1$ in log2) or too small $(<0.5$, or $<-1$ in log2) for initiating 2D base-calling (red). However, there are also base-called fast5 files with both template and complement reads where the ratio is within range for $2 \mathrm{D}$ base-calling and where $2 \mathrm{D}$ base-calling fails (grey). 


\section{Supplementary Figure 2}
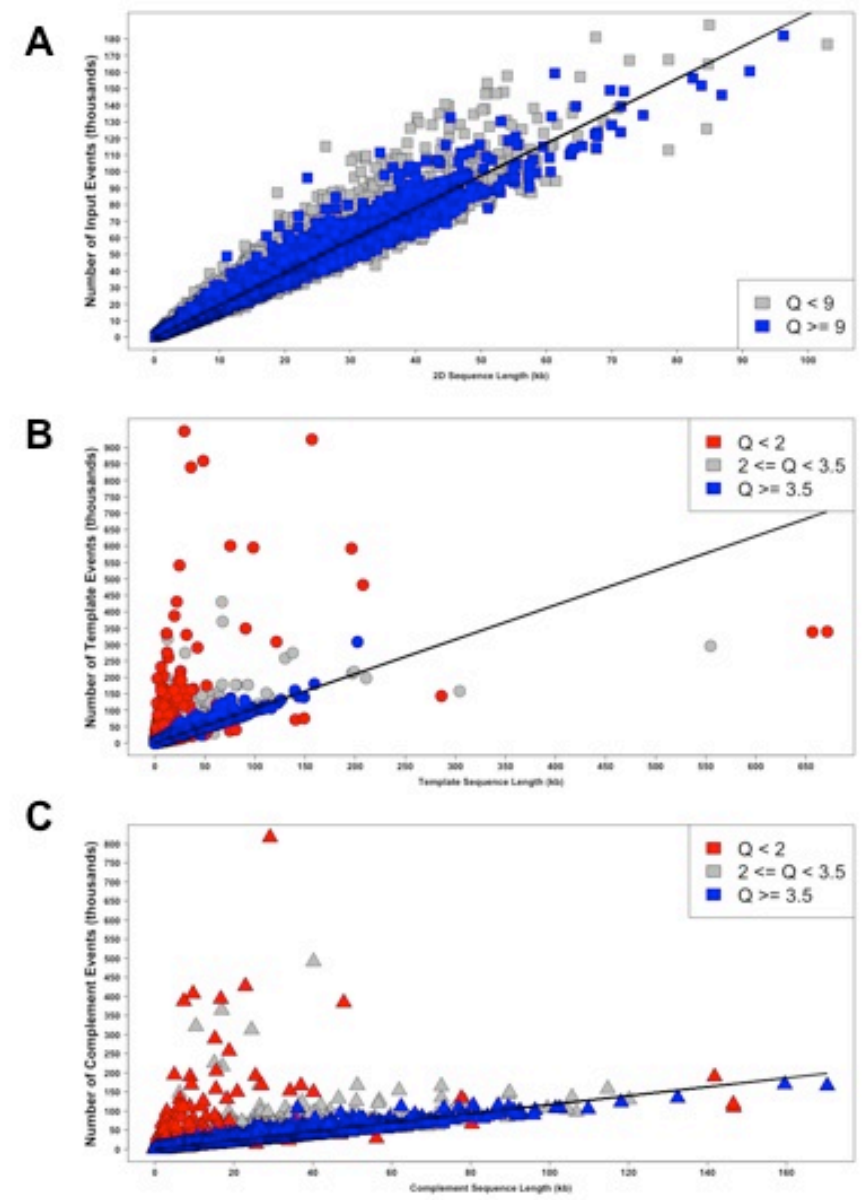

Supplementary Figure 2: Number of pre-base-calling events vs. post-base-calling sequence length.

(A) Number of total input events vs. 2D read length for reads with 2D base-calls. The slope of line is approximately $2(1.95)$ representing $\sim 2$ events per nucleotide on average for fast5 files that contain a $2 D$ read. Blue represents the high quality $(Q \geq 9) 2 D$ reads. (B) Number of template events (not to be confused with number of called template events where some are pared out) versus template sequence length. The slope (1.051) of $\sim 1$ indicates $\sim 1$ event per nucleotide on average. Blue represents higher quality 1D reads $(Q \geq 3.5)$ while red represents lower quality $1 D$ reads $(Q<2)$. (C) Number of complement events versus complement sequence lengths shows a slope of $\sim 1$ (1.166) representing $\sim 1$ event per nucleotide on average. Blue and red are as in B. Prior to base-calling, one can estimate molecule size using 1-2 events per nucleotide (depending on whether the fast5 file contains only template events, if there are also complement events, and if so, how many) noting that a minority of exceptions have higher event:nucleotide ratios. This means that 1-2 events per nucleotide is often an upper limit estimate for molecule size. Importantly, template and complement sequences with mean quality scores $>3.5$ (blue in $B$ and $C$ ) stay tightly packed around the $\sim 1$ event:nt line whereas those with $Q<3.5$ (grey and red in $B$ and $C$ ) often fall off the line (especially when $Q<2$; red), demonstrating that using $Q>3.5$ as a cut-off for $1 D$ reads has a practical interpretation that may be useful. 


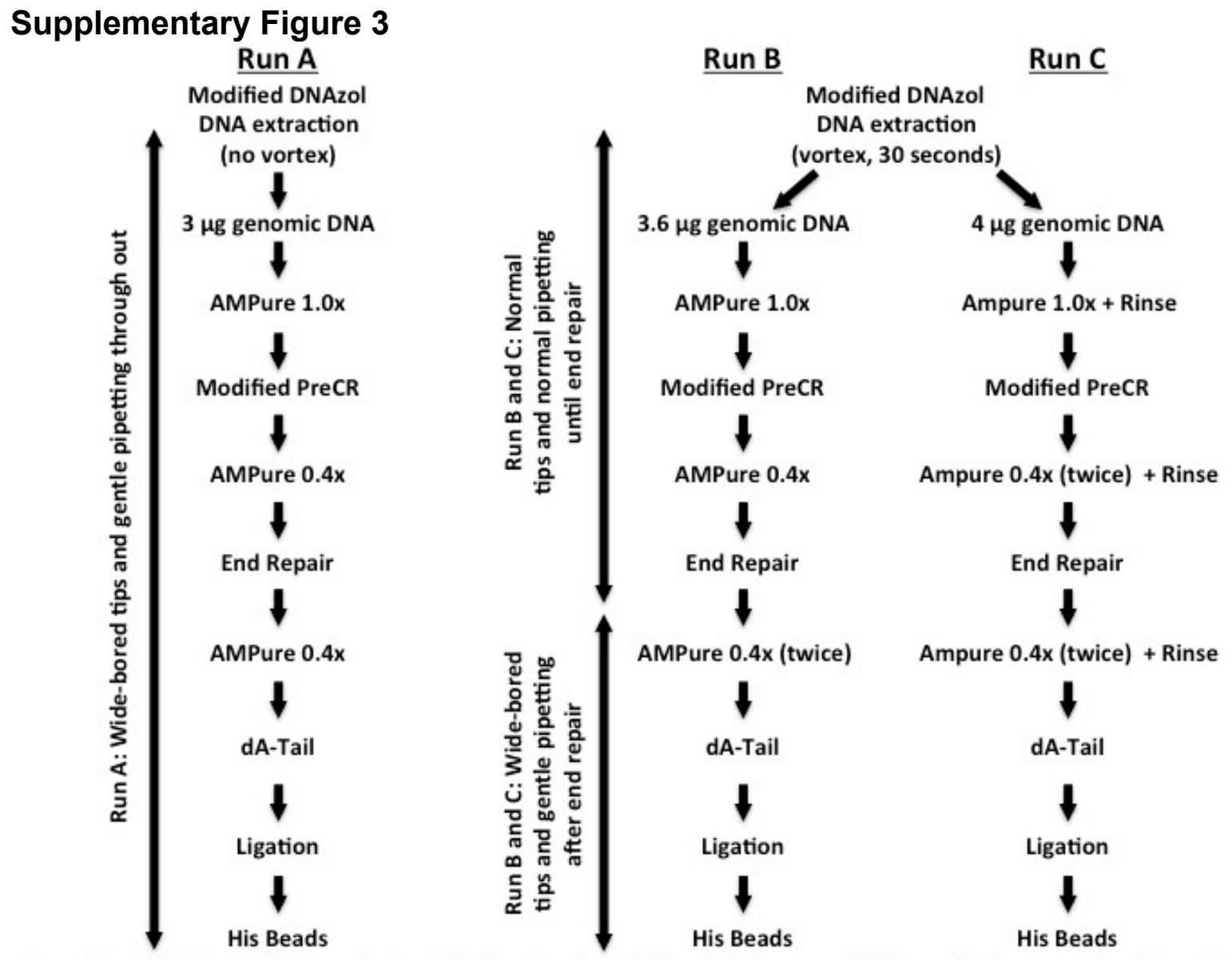

Runs $A, B$, and $C$ all used 20 minute elutions off AMPure beads at $37^{\circ} \mathrm{C}$ for all AMPure steps. AMPure ratios always performed in minimum of 100 ul total volume. DNA for all runs was prepared fresh, never frozen, and never brought above $37^{\circ} \mathrm{C}$.

\section{Supplementary Figure 3:}

See Supplementary Methods for more information. Briefly, for Run A, we gently let freshly obtained precipitated DNA re-suspend in TE (pH 8.0), skipped the Covaris shearing step in the standard protocol, used wide-bored tips with gentle pipetting to minimize DNA breakage, and started with $3 X$ the recommended starting material $(3 \mu \mathrm{g}$ instead of $1 \mu \mathrm{g}$ ) to compensate for differences in molarity. PreCR was done in double the volume with double the reagents for double the time since we started with more DNA than recommended. Both AMPure steps after PreCR were done at $0.4 \mathrm{x}$. For Run $B$, we vortexed the DNA (full speed, 30 seconds) after DNA extraction and used normal pipette tips until end-repair, after which wide-bored tips and gentler pipetting were employed. Moreover, to account for the possible increase of molecules $<1 \mathrm{~kb}$ due to vortexing, we started with more material (3.6x) and performed two sequential $0.4 x$ AMPure bead clean-ups after end-repair. For Run C, we used $4 x$ the recommended input amount of DNA, did two sequential 0.4x AMPure clean-ups before and two after end-repair, and did a new rinse step (see Supplementary Methods and Supplementary Fig 4B) at the end of all AMPure steps before the final elution of DNA off the beads. In the rinse, smaller DNA preferentially falls off the beads. For all runs, in all AMPure elutions, the beads were incubated at $37^{\circ} \mathrm{C}$ for $\geq 20$ minutes to facilitate and wait for long DNA to come off the beads. Also, DNA was never subject to temperature extremes below $4^{\circ} \mathrm{C}$ or above $37^{\circ} \mathrm{C}$ and was re-suspended in $1 \mathrm{X} \mathrm{TE}, \mathrm{pH} 8$ when isolated. 
bioRxiv preprint doi: https://doi.org/101101/019281; this version posted June 22, 2015 . The copyright holder for this preprint (which was not certified by peer review) is the author/funder, who has granted bioRxiv a license to display the preprint in perpetuity. It is made available under aCC-BY-NC 4.0 International license.

Moreover, in AMPure bead steps, the ethanol washes were performed with buffered $80 \%$ ethanol (10 mM Tris- $\mathrm{Cl}, \mathrm{pH} 8$ ). 


\section{Supplementary Figure 4}

A

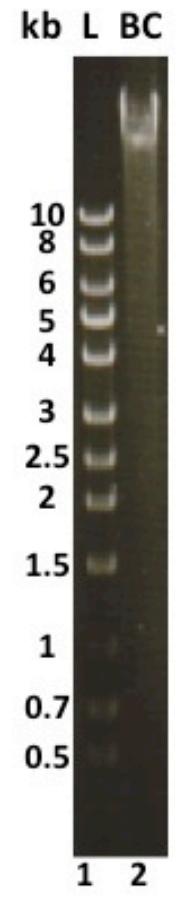

- 2010510 - -

B

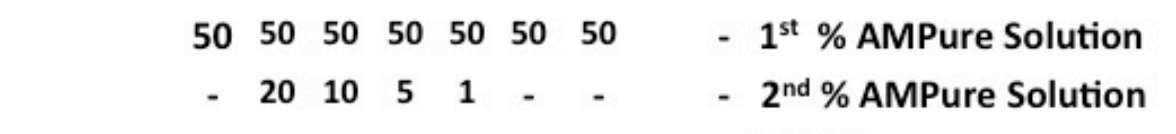

- $1^{\text {st }} \%$ AMPure Solution

- $2^{\text {nd }} \%$ AMPure Solution

\section{Supplementary Figure 4:}

(A) " $\mathrm{L}$ " is the DNA ladder and "kb" shows the sizes of the bands in the ladder. "BC" is the genomic DNA used for both Run B and Run C. Vortexing was used to help resuspend the DNA after extraction. This gel shows that a substantial proportion remains above $10 \mathrm{~kb}$ nonetheless.

(B) "L" and "kb" same as in A. Genomic DNA was gently extracted and re-suspended. Eight $15 \mu \mathrm{l}$ aliquots were set aside. The rest was lightly sonicated/sheared using the BioRuptor (Diagenode) to a very broad range with a lower limit near $0.5 \mathrm{~kb}$ and an upper limit at the size of unsheared DNA (unsheared DNA shown in lane 10). Equal volumes of sheared DNA were added to 7 of the 8 aliquots (lanes 2-8, not lane 10). We then tried several strategies to deplete the shorter DNA (e.g. $<10 \mathrm{~kb}$ ), all using AMPure beads (Lanes 3-8). Lane 2 shows the sheared+unsheared DNA without depletion of smaller DNA. Each of the aliquots with sheared DNA added (Lanes 2-8) was brought to $100 \mu \mathrm{l}$ volume with Ultra Pure Water (UPW, Life Technologies UltraPure DNase/RNase-free, distilled water). $100 \mu \mathrm{l}$ AMPure beads were then added to each to create a $50 \%$ AMPure mixture (or 1.0x ratio). The DNA was incubated in the $50 \%$ AMPure solution for 5 minutes before pelleting the beads on a magnet for 5 minutes and removing the supernatant. The beads were then washed twice with $80 \%$ ethanol while on the magnet. After the second $80 \%$ ethanol wash, the tubes with the beads were lightly spun and placed on the magnetic rack for 1 minute before removing the remaining $80 \%$ ethanol collected at the bottom from the light spin. The previous steps were performed to remove any buffers or salts associated with the DNA to have finer control over the AMPure ratio (for lanes 3-6) in subsequent steps. The beads from lane 2 were air-dried 
and eluted at this point. For lanes 3-8, instead of eluting, the beads were re-suspended in a second AMPure solution or "rinsed" once (lane 7) or twice (lane 8) before eluting. For lanes 3-6, AMPure solutions were premade by combining $40 \mu$ AMPure with $160 \mu$ l UPW (20\%, lane 3), $20 \mu$ l AMPure with $180 \mu$ UPW (10\%, lane 4), $10 \mu$ I APure with $190 \mu \mathrm{l}$ UPW (5\%, lane 5), and $2 \mu \mathrm{l}$ AMPure with $198 \mu \mathrm{l}$ UPW (1\%, lane 6). In all cases (lanes 3-6), the second AMPure solution was added to the beads from the first AMPure step (after the $80 \%$ ethanol washes described above), and the beads were gently resuspended, incubated for 10 minutes, and put on a magnetic rack for 5 minutes before the supernatant was removed and two $80 \%$ ethanol washes were performed while the beads remained on the rack. After the second $80 \%$ ethanol wash, the tubes with the beads were lightly spun and placed on the magnetic rack for 1 minute before removing the remaining $80 \%$ ethanol collected at the bottom from the light spin. The tubes were then allowed to air dry for 2-3 minutes. For lanes 7-8, after the first set of $80 \%$ ethanol washes, instead of proceeding to a second AMPure step, the tubes with the beads were lightly spun and placed on the magnetic rack for 1 minute before removing the remaining $80 \%$ ethanol collected at the bottom from the light spin. The tubes were then allowed to air dry on the magnetic rack for 2 minutes before adding $200 \mu$ UPW very gently to the tube-wall opposite the beads while they remained on the magnetic rack. This is the "rinse". The beads were allowed to incubate in the rinse for 1 minute before very gently removing it. Lane 8 was subject to a second identical rinse, which seems to offer minimal additional benefits beyond the first rinse (compare lanes 7 and 8). For all elutions (lanes 2-8), DNA was eluted off the AMPure beads into $15 \mu \mathrm{LPW}$ at $37^{\circ} \mathrm{C}$ for 20 minutes to facilitate elution of long DNA. Loading buffer was added to each sample (lanes 2-8,10). The DNA samples were then loaded onto the gel, electrophoresed, and stained with ethidium bromide for visualization. Although all conditions eliminated most of the smaller DNA (e.g. $<10 \mathrm{~kb}$ ), it was possible to tell where the tails of the smears in each lane ended using longer exposure times (data not shown). Lane 3 (20\% AMPure) ended around $1 \mathrm{~kb}$, Lane 4 (10\% AMPure) ended above $2 \mathrm{~kb}$, Lane 5 (5\% AMPure) ended above $3 \mathrm{~kb}$, Lane 6 (1\% AMPure) ended above $5 \mathrm{~kb}$, Lane 7 (1 rinse) ended above $1 \mathrm{~kb}$, and Lane 8 (2 rinses) ended above $1.5 \mathrm{~kb}$. While some of the lower percentage AMPure solutions performed better than the "rinse" as judged by where the tail ended, there were also comparable losses of the large DNA (compare lanes 3-6 to lane 2). In contrast, the rinses largely removed the small DNA while retaining the majority of the large DNA (compare lanes 7-8 with lane 2).

These results were used to inform us how to modify the AMPure steps for Run C. To deplete DNA <10kb in Run C, we chose to (1) keep the strategy of sequential AMPure washes as done here (Lanes 3-6), but with the ONT recommended 0.4x AMPure ratio ( 28-29\% AMPure solution) in the first and second sequential steps instead of the $50 \%$ (1x) AMPure solution used in the first step and lower percent (1-20\%) solutions used in the second step here and (2) add a rinse step after the second sequential AMPure step. This was successful as determined by comparing the read lengths from Runs B and C, which came from the same source of DNA (Fig. 1D, Supplementary Fig. 5). 


\section{Supplementary Figure 5}

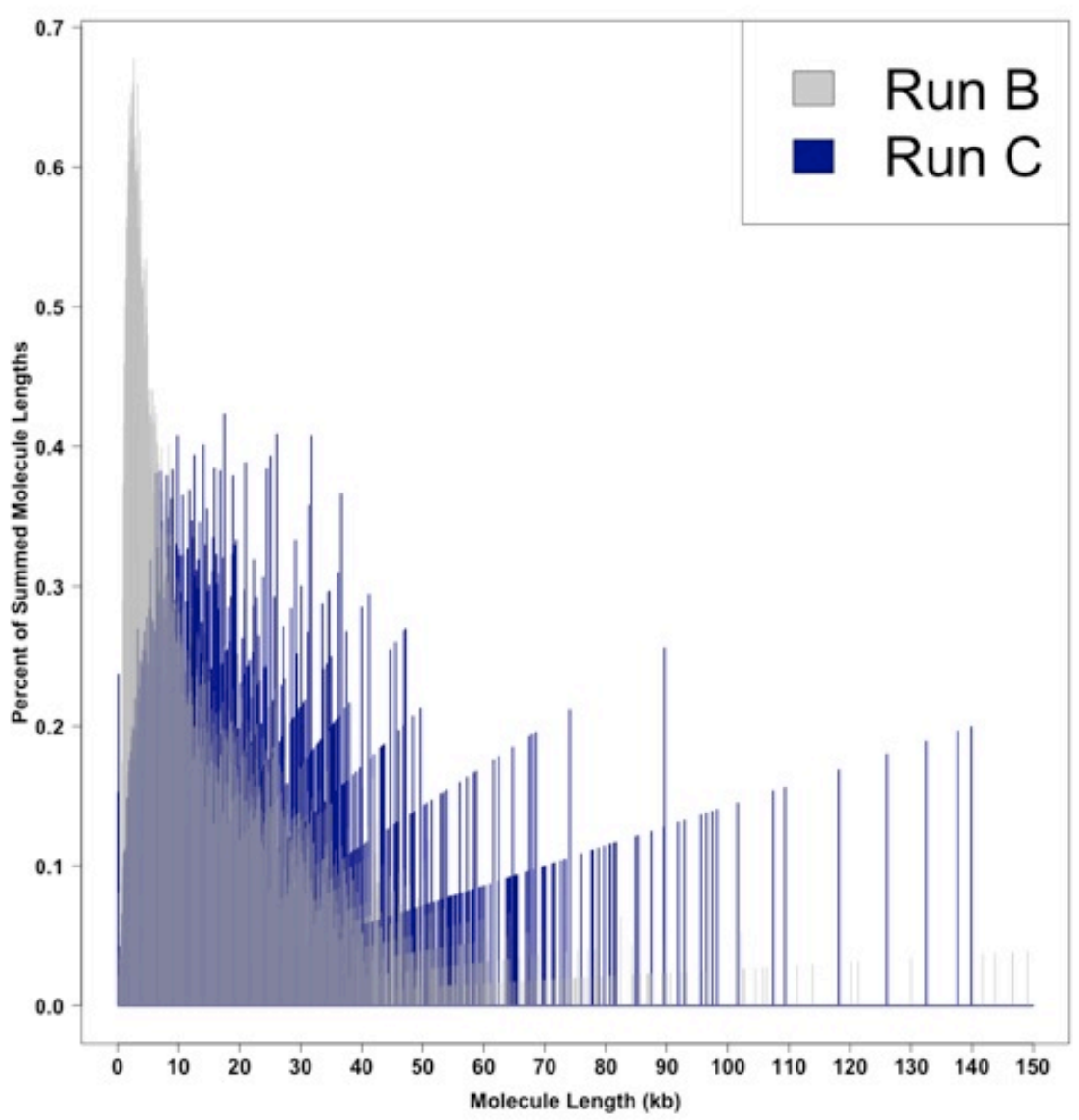

Supplementary Figure 5: The proportion of total summed molecule length as a function of molecule length for Run B (transparent grey) and Run C (dark blue). Note that the darker shade of grey is a result of the dark blue behind the transparent grey. Run B and Run C used the same source of DNA, but differed in library preparation (see Supplementary Fig. 3). Run C used a new rinse step during all AMPure clean-ups as well as took additional advantage of sequential AMPure rounds in each clean-up step. 


\section{Supplementary Figure 6}

A

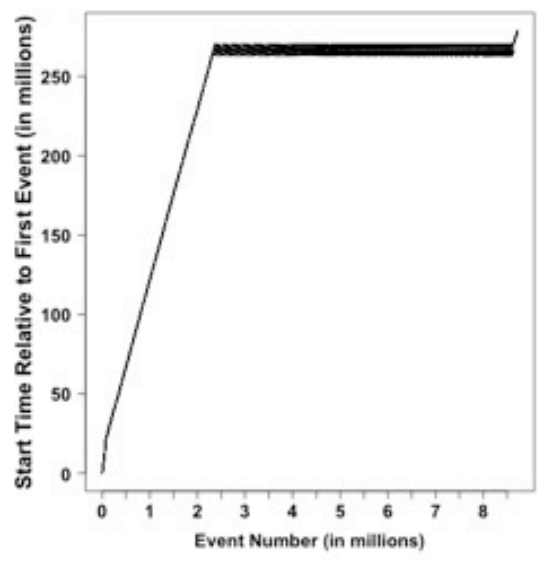

C

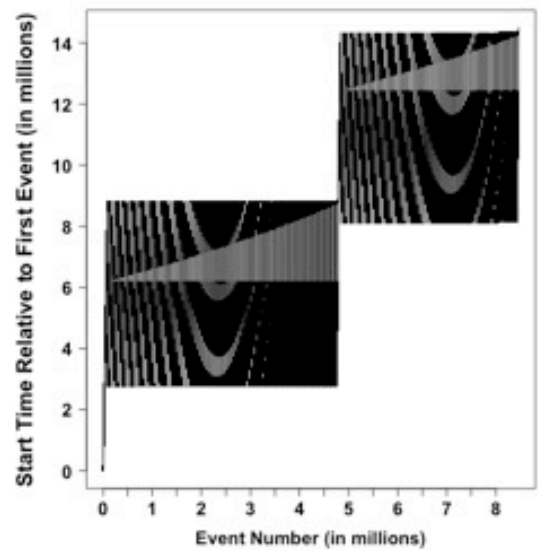

B

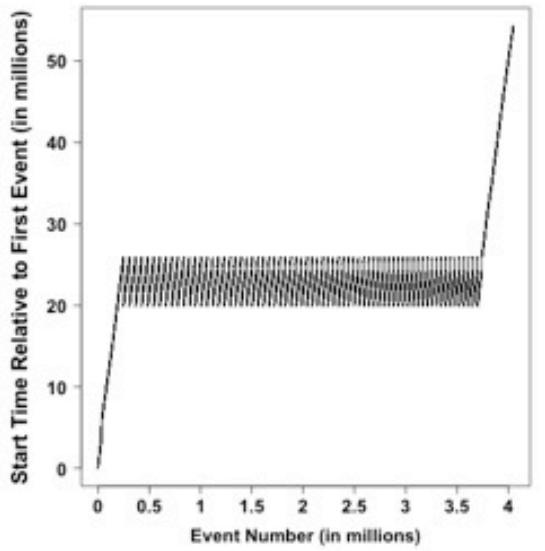

D

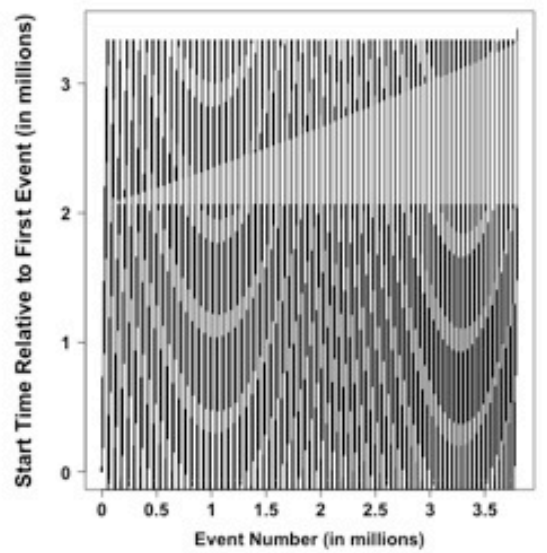

Supplementary Figure 6: Examples of multi-million event files that contained "Time Errors" (repeated blocks of events).

(A) Example from Run A.

(B) Another example from Run A.

(C) Example from Run B.

(D) Example from Run C.

The "event start time" as a function of "event number" should be a monotonically increasing function. However, in files with repeated blocks of events, the function is not monotonically increasing and instead exhibits periodic patterns. 


\section{Supplementary Figure 7}

A

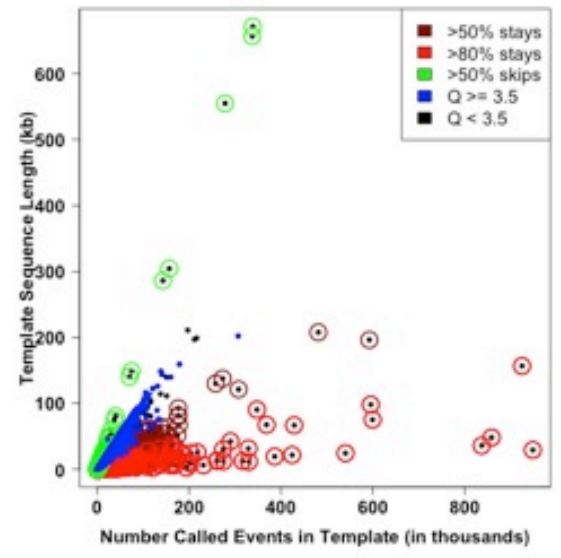

C

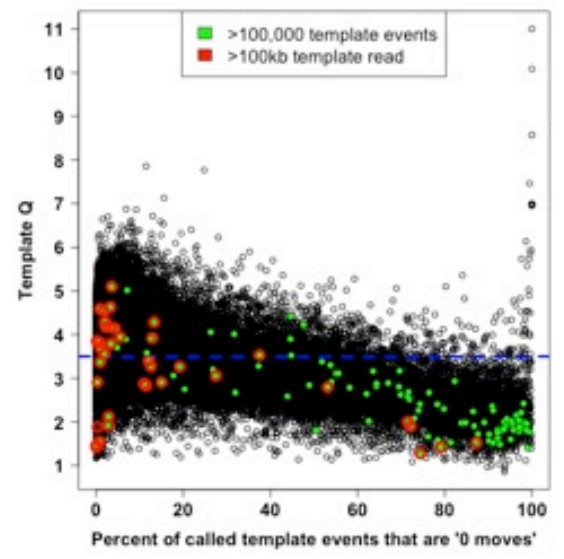

B

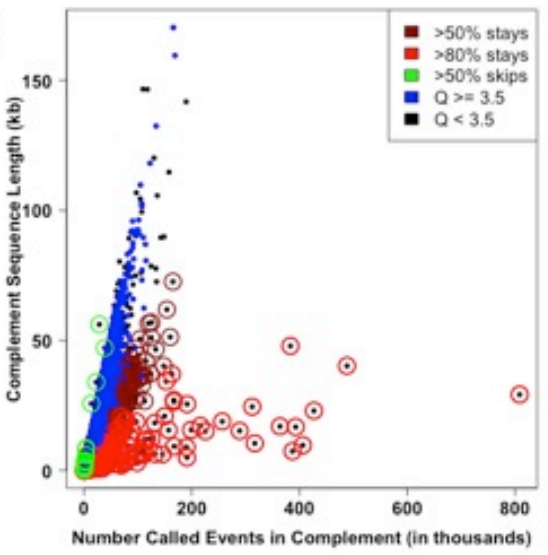

D

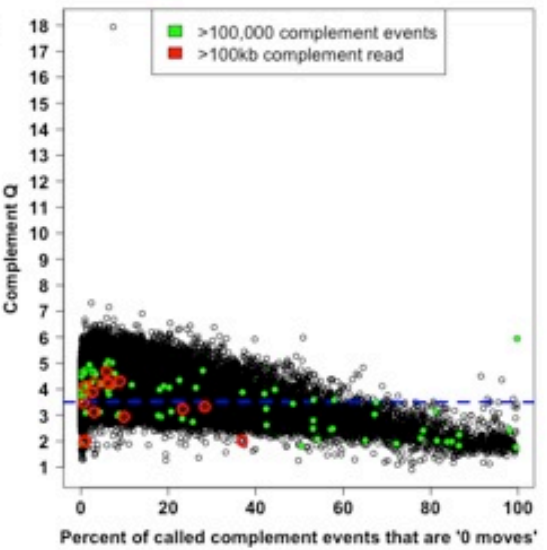

Supplementary Figure 7: Number of base-called events, sequence lengths, percent of base-called events assigned " 0 moves", and mean quality scores.

(A) Number of base-called template events vs. template sequence length with information on mean quality scores and percent of base-called template events assigned a "0 move" ("stay"). (B) Number of base-called complement events vs. complement sequence length with information on mean quality scores and percent of base-called complement events assigned a "0 move" ("stay"). (C) Percent of called template events assigned a "0 move" (stay) vs. mean quality score (Q). Slope of best fit line $(\operatorname{Im}(y \sim x)$ in $R)$ is -0.01713 meaning for every 1 percent (or 10 percent) increase in " 0 moves" there is an average decrement of 0.01713 (or 0.1713 ) in Q. (D) Percent of called complement events assigned a "0 move" (stay) vs. mean quality score (Q). Slope of best fit line $(\operatorname{lm}(y \sim x)$ in $R)$ is -0.02363 meaning for every 1 percent (or 10 percent) increase in "0 moves" there is an average decrement of 0.02363 (or 0.2363 ) in Q.

In $(A)$ and $(C)$, higher quality 1D reads $(Q \geq 3.5)$ are blue and those less than that are black. Maroon circles are around points with $>50 \%$ of base-called events assigned a " 0 move", red circles are around points with $\geq 80 \%$ of base-called events assigned a " 0 move", and green circles are around points where $>50 \%$ of the base-called events were "skips" (move=2). In (C) and (D), the blue dashed line is at $Q=3.5$, large green points represent points with $>100,000$ called template (C) or complement (D) events and maroon circles encompass points that had $\geq 100 \mathrm{~kb}$ template (C) or complement (D) sequences. 


\section{Supplementary Figure 8}
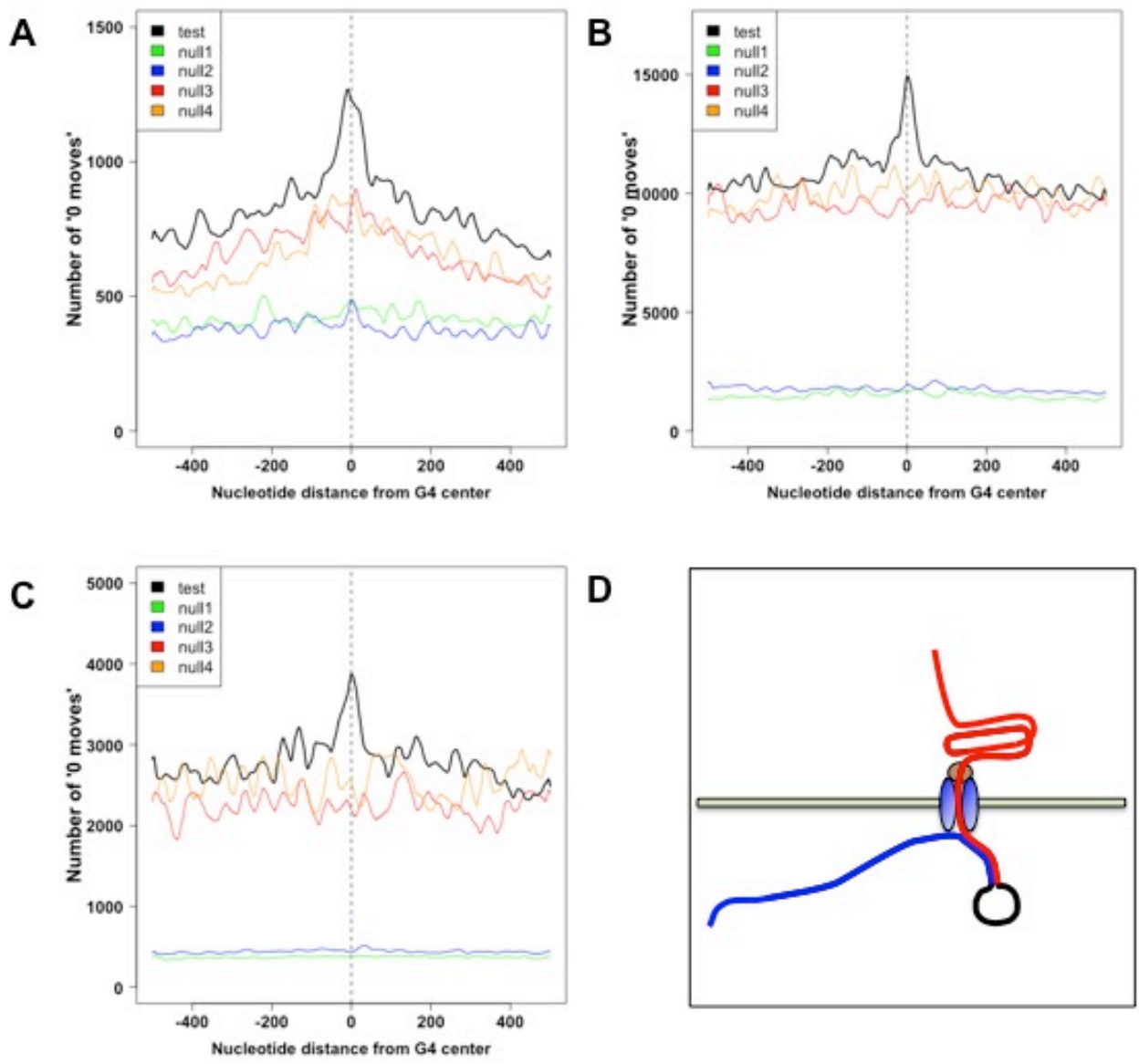

\section{Supplementary Figure 8: Aggregate analyses of the distribution of "0 moves" around $\mathbf{G} 4$ motif centers.}

The number of " 0 moves" as a function of proximity to G4 motif centers (test condition) or the centers of randomly selected positions (null 1-4 conditions) for (A) Run A, (B) Run B, and (C) Run C. (D) Depicts the orientation (5'-3') of a DNA molecule going through a pore from the top chamber to the bottom chamber and how a G4 might cause a DNA molecule to stall resulting in an accumulation of measurements of a $5 \mathrm{mer}$ or set of 5 mers slightly upstream of the G4 (the adjacent upstream sequence is pulled through the pore before the downstream G4 blocks further translocation). The template strand is blue, the hairpin is black, and the complement strand is red. 


\section{Supplementary Tables}

Supplementary Table 1A: Statistics on Mean Quality Scores (Q) for 2D and 1D reads from Run $A$

\begin{tabular}{|c|c|c|c|c|}
\hline Run A & 2D & 1D & Template & Complement \\
\hline Median Q & 9.23666606763 & 3.54781658091 & 3.41144557734 & 4.0100659241 \\
\hline Mean Q & 9.13260594983 & 3.553805971 & 3.43220450158 & 3.93551548089 \\
\hline $\begin{array}{c}\text { Standard } \\
\text { Deviation of } \\
\text { Q }\end{array}$ & 1.08997018784 & 0.916461481548 & 0.871848558243 & 0.947880924972 \\
\hline Minimum Q & 5.79424190524 & 0.965373861239 & 0.965373861239 & 1.12938538308 \\
\hline Maximum Q & 12.2263387187 & 10.0869987652 & 10.0869987652 & 6.43558322934 \\
\hline
\end{tabular}

The group of 1D reads contains all template and complement reads.

Supplementary Table 1B: Statistics on Mean Quality Scores (Q) for 2D and 1D reads from Run $B$

\begin{tabular}{|c|c|c|c|c|}
\hline Run B & 2D & 1D & Template & Complement \\
\hline Median Q & 8.59116706044 & 3.77054238697 & 3.58182241161 & 4.21229580534 \\
\hline Mean Q & 8.61912781671 & 3.80409144806 & 3.59890226341 & 4.1571515184 \\
\hline $\begin{array}{c}\text { Standard } \\
\text { Deviation of } \\
\text { Q }\end{array}$ & 0.988906885917 & 0.782651340952 & 0.691010548999 & 0.804879951209 \\
\hline Minimum Q & 4.51060190561 & 0.855017497526 & 0.855017497526 & 0.892928322743 \\
\hline Maximum Q & 12.9319853199 & 11.0 & 11.0 & 7.31138333836 \\
\hline
\end{tabular}

The group of 1D reads contains all template and complement reads.

Supplementary Table 1C: Statistics on Mean Quality Scores (Q) for 2D and 1D reads from Run C

\begin{tabular}{|c|c|c|c|c|}
\hline Run C & 2D & 1D & Template & Complement \\
\hline Median Q & 9.07294962204 & 3.79287980744 & 3.62309975937 & 4.13185844371 \\
\hline Mean Q & 8.96810671735 & 3.73547257167 & 3.60595627604 & 4.05290820742 \\
\hline $\begin{array}{c}\text { Standard } \\
\text { Deviation of } \\
\text { Q }\end{array}$ & 0.976642865308 & 0.899676295249 & 0.872084862604 & 0.887443696552 \\
\hline Minimum Q & 4.95929992076 & 1.11272829123 & 1.11272829123 & 1.16860148363 \\
\hline Maximum Q & 12.9534046975 & 17.9360409474 & 7.46183460838 & 17.9360409474 \\
\hline
\end{tabular}

The group of 1D reads contains all template and complement reads. 
Supplementary Table 2: Read types in base-called fast5 files.

\begin{tabular}{|l|l|l|l|}
\hline & $\begin{array}{l}\text { Run } \\
\text { A }\end{array}$ & $\begin{array}{l}\text { Run } \\
\text { B }\end{array}$ & $\begin{array}{l}\text { Run } \\
\text { C }\end{array}$ \\
\hline n_molecules (\# basecalled files) & 9935 & 64282 & 8941 \\
\hline n_template_only & 6770 & 26923 & 5293 \\
\hline percent_template_only & 68.14 & 41.88 & 59.20 \\
\hline n_has_complement & 3165 & 37359 & 3648 \\
\hline percent_has_complement & 31.86 & 58.12 & 40.80 \\
\hline n_has_2D & 2172 & 31999 & 2523 \\
\hline percent_has_2D & 21.86 & 49.78 & 28.22 \\
\hline n_does_NOT_have_2D & 7763 & 32283 & 6418 \\
\hline percent_does_NOT_have_2D & 78.14 & 50.22 & 71.78 \\
\hline $\begin{array}{l}\text { percent_of_molecules_that_do_NOT_have_2D } \\
\text { because_template_only }\end{array}$ & 87.21 & 83.40 & 82.47 \\
\hline $\begin{array}{l}\text { percent_of_molecules_that do_NOT_have_2D_that } \\
\text { DO_have_comlement }\end{array}$ & 12.79 & 16.60 & 17.53 \\
\hline n_with_complement_that_DO_have_2D & & & \\
\hline n_with_complement_that_do_NOT_have_2D & 2172 & 31999 & 2523 \\
\hline percent_of_molecules_with_complement that_DO_have_2D & 993 & 5360 & 1125 \\
\hline percent_of_molecules_with_complement_that_do_NOT_have_2D & 68.63 & 85.65 & 69.16 \\
\hline & & & \\
\hline
\end{tabular}




\section{Supplementary Table 3: Molecule and Read Length Statistics}

All lengths are nucleotide lengths in Supplementary Tables $3 A-E$ (e.g. 10,000 = 10,000 nucleotides).

\section{Supplementary Table 3A: Molecule Statistics}

\begin{tabular}{|l|l|l|l|}
\hline & Run A & Run B & Run C \\
\hline sum_molecule_lengths & $49,778,211$ & $386,880,692$ & $70,086,870$ \\
\hline Molecule N25 & 46,973 & 28,006 & 34,939 \\
\hline Molecule N50 & 25,238 & 13,553 & 20,824 \\
\hline Molecule N75 & 11,969 & 5,279 & 10,749 \\
\hline mean_molecule_size & $5,010.4$ & $6,018.5$ & $7,838.8$ \\
\hline median_molecule_size & 152 & 2,817 & 3,116 \\
\hline max_molecule_size & 304,309 & 671,219 & 139,864 \\
\hline min_molecule_size & 5 & 5 & 5 \\
\hline n_molecules_gt_10kb & 1,433 & 10,145 & 2,318 \\
\hline percent_molecules_gt_10kb & 14.42 & 15.78 & 25.93 \\
\hline sum_molecules_gt_10kb & $39,415,998$ & $226,507,295$ & $54,080,719$ \\
\hline percent_summed_molecules_from_molecules_gt_10kb & 79.18 & 58.55 & 77.16 \\
\hline n_molecules_gt_50kb & 139 & 396 & 119 \\
\hline percent_molecules_gt_50kb & 1.40 & 0.62 & 1.33 \\
\hline sum_molecules_gt_50kb & $10,999,924$ & $27,504,214$ & $8,168,033$ \\
\hline percent_summed_molecules_from_molecules_gt_50kb & 22.10 & 7.11 & 11.65 \\
\hline n_molecules_gt_100kb & 21 & 24 & 8 \\
\hline percent_molecules_gt_100kb & 0.21 & 0.04 & 0.09 \\
\hline sum_molecules_gt_100kb & $3,140,720$ & $4,779,702$ & 972,486 \\
\hline percent_summed_molecules_from_molecules_gt_100kb & 6.31 & 1.24 & 1.39 \\
\hline & & & \\
\hline
\end{tabular}

Molecule size is determined as (1) the length of the 2D read if one is available, (2) the length of the template if only the template is available, or (3) the longer of the template and complement reads when both are available in the absence of a 2D read (See supplementary methods). Thus, the statistics are performed on a set of unique/nonredundant molecule length estimates (a single read for each molecule as opposed to 13 reads per molecule).

Supplementary Table 3B: 2D Read Statistics

\begin{tabular}{|l|l|l|l|}
\hline & Run A & Run B & Run C \\
\hline sum_HQ_(Q>=9)_2d_lengths & 13220898 & 74311821 & 15790614 \\
\hline HQ_2D N25 & 34893 & 23192 & 30278 \\
\hline HQ_2D N50 & 18145 & 11620 & 18008 \\
\hline HQ_2D N75 & 9002 & 5194 & 10013 \\
\hline mean_HQ_2d_length & 10722.5 & 6519.72 & 11593.7 \\
\hline median_HQ_2d_length & 6393 & 3580 & 8061.5 \\
\hline sum_ALL_2d_lengths & 24017633 & 188595516 & 27734513 \\
\hline ALL 2D N25 & 37314 & 23647 & 31130 \\
\hline ALL 2D N50 & 19460 & 11200 & 18426 \\
\hline
\end{tabular}


bioRxiv preprint doi: https://doi.org/10.1101/019281; this version posted June 22, 2015. The copyright holder for this preprint (which was not certified by peer review) is the author/funder, who has granted bioRxiv a license to display the preprint in perpetuity. It is made available under aCC-BY-NC 4.0 International license.

\begin{tabular}{|l|l|l|l|}
\hline ALL 2D N75 & 9614 & 4681 & 9801 \\
\hline mean_2d_length & 11057.8 & 5893.8 & 10992.7 \\
\hline median_2d_length & 6356 & 3051 & 7210 \\
\hline min_2d_length (Q) & $94(6.33)$ & $81(6.51)$ & $82(5.97)$ \\
\hline max_2d_length (Q) & $102935(8.74)$ & $86797(9.01)$ & $84898(8.87)$ \\
\hline max_2d_length_Q_ge_9 & $96237(9.61)$ & $86797(9.01)$ & $71380(9.04)$ \\
\hline max_2d_length_Q_ge_8.5 & $102935(8.74)$ & $86797(9.01)$ & $84898(8.87)$ \\
\hline max_2d_length_Q_ge_8 & $102935(8.74)$ & $86797(9.01)$ & $84898(8.87)$ \\
\hline max_2d_length_Q_ge_7.5 & $102935(8.74)$ & $86797(9.01)$ & $84898(8.87)$ \\
\hline
\end{tabular}

Supplementary Table 3C: 1D Read Statistics.

\begin{tabular}{|l|l|l|l|}
\hline & Run A & Run B & Run C \\
\hline sum_1d_lengths & 75870350 & 565275221 & 102225612 \\
\hline 1D N25 & 41287 & 25353 & 33308 \\
\hline 1D N50 & 21927 & 12037 & 19812 \\
\hline 1D N75 & 10432 & 4776 & 10349 \\
\hline mean_1d_length & 5791.6 & 5561.5 & 8120.2 \\
\hline median_1d_length & 231 & 2661 & 3783 \\
\hline & & & \\
\hline sum_template_lengths & 43984348 & 349320608 & 66858648 \\
\hline Template N25 & 43176 & 25699 & 33509 \\
\hline Template N50 & 22324 & 12198 & 20001 \\
\hline Template N75 & 10344 & 4752 & 10421 \\
\hline mean_template_length & 4427.2 & 5434.2 & 7477.8 \\
\hline median_template_length & 151 & 2565.5 & 2892 \\
\hline min_template_length (Q) & $5(10.09)$ & $5(6.95)$ & $5(6.97)$ \\
\hline max_template_length & $304309(2.12)$ & $671219(1.55)$ & $139864(4.28)$ \\
\hline max_template_Q_ge_4_length & $122689(4.63)$ & $143763(4.17)$ & $139864(4.28)$ \\
\hline max_template_Q_ge_3.5_length & $202293(3.53)$ & $143763(4.17)$ & $139864(4.28)$ \\
\hline max_template_Q_ge_3_length & $210931(3.36)$ & $143763(4.17)$ & $139864(4.28)$ \\
\hline max_template_Q_ge_2.5_length & $210931(3.36)$ & $554720(2.91)$ & $139864(4.28)$ \\
\hline & & & \\
\hline sum_complement_lengths & 31886002 & 215954613 & 35366964 \\
\hline Complement N25 & 39391 & 24784 & 33054 \\
\hline Complement N50 & 21394 & 11821 & 19287 \\
\hline Complement N75 & 10506 & 4825 & 10163 \\
\hline mean_complement_length & 10074.6 & 5780.5 & 9694.9 \\
\hline median_complement_length & 5049 & 2836 & 5700 \\
\hline min_complement_length & $17(1.13)$ & $14(3.63)$ & $10(4.36)$ \\
\hline max_complement_length & $170334(3.87)$ & $146631(2.00)$ & $132439(4.20)$ \\
\hline max_complement_Q_ge_4_length & $159563(4.30)$ & $102671(4.20)$ & $132439(4.20)$ \\
\hline max_complement_Q_ge_3.5_length & $170334(3.87)$ & $102671(4.20)$ & $132439(4.20)$ \\
\hline max_complement_Q_ge_3_length & $170334(3.87)$ & $105683(3.23)$ & $132439(4.20)$ \\
\hline max_complement_Q_ge_2.5_length & $170334(3.87)$ & $120149(2.94)$ & $132439(4.20)$ \\
\hline & & & \\
\hline
\end{tabular}

Note: Statistics on "1D reads" are computed on all template and complement reads together (rather than treating the two read types separately). 


\section{Supplementary Table 3D: Top 10 2D lengths with mean quality score (Q) across} all 3 runs

\begin{tabular}{|l|l|l|l|}
\hline Rank & Length & Q & Run \\
\hline 1 & 102935 & 8.74 & A \\
\hline 2 & 96237 & 9.61 & A \\
\hline 3 & 91062 & 9.93 & B \\
\hline 4 & 86797 & 9.01 & C \\
\hline 5 & 84898 & 8.87 & A \\
\hline 6 & 84716 & 8.28 & B \\
\hline 7 & 84477 & 7.75 & A \\
\hline 8 & 83697 & 9.34 & B \\
\hline 9 & 82373 & 9.73 & A \\
\hline 10 & 78658 & 8.24 & A \\
\hline
\end{tabular}

Supplementary Table 3E: Top $101 \mathrm{D}$ reads where mean quality $\mathrm{Q}>3$

\begin{tabular}{|l|l|l|l|l|}
\hline Rank & Length & Q & Run & Read Type \\
\hline 1 & 210931 & 3.37 & A & Template \\
\hline 2 & 202293 & 3.53 & A & Template \\
\hline 3 & 199014 & 3.40 & A & Template \\
\hline 4 & 170334 & 3.87 & A & Complement \\
\hline 5 & 159563 & 4.30 & A & Complement \\
\hline 6 & 159397 & 3.92 & A & Template \\
\hline 7 & 148912 & 3.70 & A & Template \\
\hline 8 & 143763 & 4.17 & B & Template \\
\hline 9 & 139864 & 4.28 & C & Template \\
\hline 10 & 132439 & 4.20 & C & Complement \\
\hline
\end{tabular}


Supplementary Table 4A: Number of pores available for sequencing.

\begin{tabular}{|c|c|c|c|}
\hline $\begin{array}{c}\text { Estimated number } \\
\text { of channels } \\
\text { available at QC }\end{array}$ & 191 & Run A & Run C \\
\hline $\begin{array}{c}\text { Number of } \\
\text { channels that had } \\
\text { at least 1 read } \\
\text { throughout run }\end{array}$ & 214 & 491 & 448 \\
\hline
\end{tabular}

Supplementary Table 4B: Schedule and recipes for adding sequencing mixes to flow cells during MinION sequencing.

\begin{tabular}{|c|c|c|c|c|c|c|c|c|c|c|c|c|}
\hline & \multicolumn{4}{|c|}{ Run A } & \multicolumn{4}{|c|}{ Run B } & \multicolumn{4}{|c|}{ Run C } \\
\hline & PSM & EP & Fuel & HAP & PSM & EP & Fuel & HAP & PSM & EP & Fuel & HAP \\
\hline SM1 & 6 & 140 & 4 & 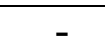 & 3 & 144 & 3 & - & 6 & 140 & 4 & - \\
\hline SM2 & 6 & 140 & 4 & 10 & 4 & 142 & 3 & 1 & 6 & 140 & 4 & 1 \\
\hline SM3 & 3 & 143 & 4 & 6 & 4 & 143 & 3 & 8 & 3 & 144 & 3 & 6 \\
\hline SM4 & 6 & 140 & 4 & 8 & 4 & 174 & 4 & 15 & 2 & 145 & 3 & 12 \\
\hline SM5 & 3 & 143 & 4 & 13 & 3 & 144 & 3 & 5.5 & 3 & 143 & 4 & 5 \\
\hline SM6 & $\mathrm{R}$ & 143 & 4 & 5 & 4 & 142 & 4 & 4 & $\mathrm{R}$ & 145 & 3 & 7 \\
\hline SM7 & - & - & - & - & $\mathrm{R}$ & 144 & 4 & 10 & - & - & - & - \\
\hline
\end{tabular}

HAP $=$ Hours After Previous (i.e. time after adding previous SM)

$\mathrm{PSM}=$ Pre-Sequencing Mix

$\mathrm{SM}=$ Sequencing Mix

$\mathrm{EP}=\mathrm{EP}$ Buffer

Fuel = "Fuel Mix" provided by ONT.

$\mathrm{R}=$ Remaining PSM

Sequencing Mix (SM) was prepared fresh immediately before adding to the flow cell at each time point.

All volumes reported for PSM, EP, and Fuel are in $\mu \mathrm{l}$. 
Supplementary Table 5: Filtering

\begin{tabular}{|c|c|c|c|}
\hline & Run A & Run B & Run C \\
\hline Number event files & & 82040 & 12536 \\
\hline Number files filtered: no template found (NTF) & $\begin{array}{l}10306 \\
(37.3 \%)\end{array}$ & $\begin{array}{c}6820 \\
(8.31 \%)\end{array}$ & $\begin{array}{c}1997 \\
(15.9 \%)\end{array}$ \\
\hline Number files filtered: too few events (TFE) & $\begin{array}{c}7414 \\
(26.8 \%)\end{array}$ & $\begin{array}{c}10878 \\
(13.26 \%)\end{array}$ & $\begin{array}{c}1583 \\
(12.6 \%)\end{array}$ \\
\hline Number of files filtered: too many events (TME) & $\begin{array}{c}9 \\
(0.03 \%)\end{array}$ & $\begin{array}{c}46 \\
(0.056 \%)\end{array}$ & $\begin{array}{c}14 \\
(0.11 \%)\end{array}$ \\
\hline of filhe filto & $\begin{array}{c}2 \\
(0.007 \%)\end{array}$ & $\begin{array}{c}14 \\
(0.017 \%)\end{array}$ & $\begin{array}{c}0 \\
(0 \%)\end{array}$ \\
\hline $\begin{array}{r}\text { Number of files } \\
\text { (percent } 0\end{array}$ & $\begin{array}{c}0 \\
(0 \%)\end{array}$ & $\begin{array}{c}0 \\
(0 \%)\end{array}$ & $\begin{array}{c}0 \\
(0 \%)\end{array}$ \\
\hline $\begin{array}{l}\text { Number of files with } 100,0 \\
\text { time error (percent of all fil }\end{array}$ & $\begin{array}{c}2 \\
(1.57 \%)\end{array}$ & $\begin{array}{c}14 \\
(5.51 \%)\end{array}$ & $\begin{array}{c}0 \\
(0 \%)\end{array}$ \\
\hline $\begin{array}{r}\text { Number of TME files } \begin{array}{r}(>1,0000 \\
\text { time er }\end{array}\end{array}$ & $\begin{array}{c}5 \text { of } 9 \\
(55.6 \%)\end{array}$ & $\begin{array}{l}30 \text { of } 46 \\
(65.2 \%)\end{array}$ & $\begin{array}{l}4 \text { of } 14 \\
(28.6 \%)\end{array}$ \\
\hline $\begin{array}{l}\text { Number of TME files ( }>1,0000,000 \text { events) that did NOT } \\
\text { have time errors }\end{array}$ & $\begin{array}{c}4 \text { of } 9 \\
(44.4 \%)\end{array}$ & $\begin{array}{l}16 \text { of } 46 \\
(34.8 \%)\end{array}$ & $\begin{array}{l}10 \text { of } 14 \\
(71.4 \%)\end{array}$ \\
\hline $\begin{array}{l}\text { Number of TME files (>1 } \\
\text { errors that did NOT }\end{array}$ & & $\begin{array}{l}6 \text { of } 16 \\
(37.5 \%)\end{array}$ & \\
\hline $\begin{array}{c}\text { Number of TME files ( }>1,0000,000 \text { events) without time } \\
\text { errors that had lead adapter profile }\end{array}$ & $\begin{array}{c}4 \text { of } 4 \\
(100 \%)\end{array}$ & $\begin{array}{c}10 \text { of } 16 \\
(62.5)\end{array}$ & $\begin{array}{l}5 \text { of } 10 \\
(50 \%)\end{array}$ \\
\hline $\begin{array}{c}\text { Number/percent of TME files ( }>1,0000,000 \text { events) that } \\
\text { passed time-error and lead adapter filtering }\end{array}$ & $\begin{array}{l}4 \text { of } 9 \\
(44.4 \%)\end{array}$ & $\begin{array}{l}10 \text { of } 46 \\
(21.7 \%)\end{array}$ & $\begin{array}{l}5 \text { of } 14 \\
(35.7 \%)\end{array}$ \\
\hline
\end{tabular}


Supplementary Table 6A: Are there more "0 moves" near G4 motifs than sites selected at random?

\begin{tabular}{|c|c|c|c|c|c|}
\hline 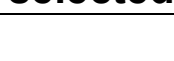 & & Null 1 & Null 2 & Null 3 & Null 4 \\
\hline \multirow{2}{*}{ Run A } & Sign & $7.554388 \mathrm{e}-26$ & $4.832767 e-38$ & $1.193051 \mathrm{e}-137$ & $5.480738 \mathrm{e}-122$ \\
\hline & \begin{tabular}{|l|} 
Signed \\
Rank
\end{tabular} & $3.090888 \mathrm{e}-27$ & $2.244648 \mathrm{e}-41$ & $4.612996 \mathrm{e}-131$ & $2.224541 \mathrm{e}-124$ \\
\hline \multirow[t]{2}{*}{ Run B } & Sign & 0 & $3.482397 e-315$ & 0 & 0 \\
\hline & \begin{tabular}{|l|} 
Signed \\
Rank
\end{tabular} & 0 & 0 & 0 & 0 \\
\hline \multirow[t]{2}{*}{ Run C } & Sign & $1.29939 \mathrm{e}-41$ & $1.698042 \mathrm{e}-36$ & $6.549949 \mathrm{e}-48$ & $3.301627 e-55$ \\
\hline & \begin{tabular}{|l|} 
Signed \\
Rank
\end{tabular} & $2.194097 e-75$ & $1.639146 \mathrm{e}-65$ & $2.938907 e-50$ & $2.956933 e-50$ \\
\hline \multirow{2}{*}{$\begin{array}{l}\text { p-value } \\
\text { product }\end{array}$} & Sign & 0 & 0 & 0 & 0 \\
\hline & \begin{tabular}{|l|} 
Signed \\
Rank
\end{tabular} & 0 & 0 & 0 & 0 \\
\hline
\end{tabular}

0 indicates that $p<1 e-324$.

G4s and stays:

G4s paired with Nulls

Null 1 = randomly selected positions on template and complement reads without G4 motifs

Null 2 = randomly selected positions on all template and complement reads

Null $3=$ randomly selected positions on the same template or complement read containing the G4 motif

Null 4 = randomly selected positions on the same template or complement read containing the G4 motif excluding positions that overlap the G4 motif

Note: $7.95 \%, 7.84 \%$, and $7.24 \%$ of Template and Complement reads contain G4 motif in Run A, Run B, and Run C, respectively.

Supplementary Table 6B: Do G4 motifs on complement strand associate with more "0 moves" than $\mathrm{G} 4$ motifs on template?

\begin{tabular}{|l|l|}
\hline & Rank Sum \\
\hline Run A & $1.033993 \mathrm{e}-30$ \\
\hline Run B & $3.952016 \mathrm{e}-26$ \\
\hline Run C & 0.004113 \\
\hline p-value product & $1.680719 \mathrm{e}-58$ \\
\hline
\end{tabular}

Supplementary Table 5C: Do G4 motifs with $>4$ tracts associate with more "0 moves"?

\begin{tabular}{|l|l|}
\hline & Rank Sum \\
\hline Run A & 0.002023 \\
\hline Run B & $2.139787 \mathrm{e}-92$ \\
\hline Run C & $4.449 \mathrm{e}-14$ \\
\hline p-value product & $1.925878 \mathrm{e}-108$ \\
\hline
\end{tabular}


Supplementary Table 6D: $Q$ score distributions for all reads, specific read types with $\geq 1 \mathrm{G} 4$ motif, any read type with $\geq 1 \mathrm{G} 4$ motif

\begin{tabular}{|c|c|c|c|c|c|c|c|}
\hline & & & $\begin{array}{c}\text { Median } \\
\mathbf{Q}\end{array}$ & $\begin{array}{c}\text { Mean } \\
\mathbf{Q}\end{array}$ & $\begin{array}{l}\text { Std } \\
\text { Dev } \\
\text { of } Q\end{array}$ & Min Q & $\operatorname{Max} \mathbf{Q}$ \\
\hline \multirow{9}{*}{$\begin{array}{c}\text { Run } \\
\mathbf{A}\end{array}$} & \multirow{3}{*}{ All reads } & 2D & 9.24 & 9.13 & 1.09 & 5.79 & 12.23 \\
\hline & & Template & 3.41 & 3.43 & 0.87 & 0.97 & 10.09 \\
\hline & & Complement & 4.01 & 3.94 & 0.95 & 1.13 & 6.44 \\
\hline & \multirow{3}{*}{$\begin{array}{l}\geq 1 \mathrm{G} 4 \text { in } \\
\text { specific } \\
\text { read type }\end{array}$} & $2 \mathrm{D}$ & 8.33 & 8.51 & 0.95 & 6.62 & 11.27 \\
\hline & & Template & 3.85 & 3.79 & 0.71 & 1.18 & 5.57 \\
\hline & & Complement & 3.81 & 3.85 & 0.77 & 1.40 & 5.47 \\
\hline & \multirow{3}{*}{$\begin{array}{c}\geq 1 \mathrm{G} 4 \text { in } \\
\text { any read } \\
\text { type }\end{array}$} & $2 \mathrm{D}$ & 8.87 & 8.88 & 1.01 & 6.62 & 11.49 \\
\hline & & Template & 3.92 & 3.87 & 0.72 & 1.18 & 10.09 \\
\hline & & Complement & 3.92 & 3.91 & 0.81 & 1.40 & 5.72 \\
\hline \multirow{9}{*}{$\begin{array}{c}\text { Run } \\
\text { B }\end{array}$} & \multirow{3}{*}{ All reads } & $2 \mathrm{D}$ & 8.59 & 8.62 & 0.99 & 4.51 & 12.93 \\
\hline & & Template & 3.58 & 3.60 & 0.69 & 0.86 & 11.0 \\
\hline & & Complement & 4.21 & 4.16 & 0.80 & 0.89 & 7.31 \\
\hline & \multirow{3}{*}{$\begin{array}{l}\geq 1 \mathrm{G} 4 \text { in } \\
\text { specific } \\
\text { read type }\end{array}$} & $2 D$ & 8.13 & 8.26 & 0.94 & 5.72 & 12.06 \\
\hline & & Template & 3.59 & 3.60 & 0.67 & 1.17 & 6.28 \\
\hline & & Complement & 3.95 & 3.97 & 0.76 & 1.36 & 6.29 \\
\hline & \multirow{3}{*}{$\begin{array}{c}\geq 1 \mathrm{G} 4 \text { in } \\
\text { any read } \\
\text { type }\end{array}$} & $2 D$ & 8.46 & 8.50 & 0.97 & 5.72 & 12.06 \\
\hline & & Template & 3.72 & 3.71 & 0.65 & 1.17 & 6.28 \\
\hline & & Complement & 3.99 & 4.00 & 0.79 & 1.10 & 6.91 \\
\hline \multirow{9}{*}{$\begin{array}{c}\text { Run } \\
\text { C }\end{array}$} & \multirow{3}{*}{ All reads } & $2 D$ & 9.07 & 8.97 & 0.98 & 4.96 & 12.95 \\
\hline & & Template & 3.62 & 3.61 & 0.87 & 1.11 & 7.46 \\
\hline & & Complement & 4.13 & 4.05 & 0.89 & 1.17 & 17.9 \\
\hline & \multirow{3}{*}{$\begin{array}{l}\geq 1 \mathrm{G} 4 \text { in } \\
\text { specific } \\
\text { read type }\end{array}$} & $2 D$ & 8.84 & 8.66 & 1.00 & 6.69 & 11.09 \\
\hline & & Template & 3.81 & 3.70 & 0.83 & 1.43 & 5.45 \\
\hline & & Complement & 3.93 & 3.83 & 0.81 & 1.18 & 5.43 \\
\hline & \multirow{3}{*}{$\begin{array}{c}\geq 1 \mathrm{G} 4 \text { in } \\
\text { any read } \\
\text { type }\end{array}$} & $2 D$ & 8.97 & 8.83 & 0.94 & 5.66 & 11.09 \\
\hline & & Template & 4.11 & 3.91 & 0.81 & 1.43 & 5.94 \\
\hline & & Complement & 4.04 & 3.96 & 0.82 & 1.18 & 5.87 \\
\hline
\end{tabular}




\section{Supplementary References}

1. Team, R. C. R: A language and environment for statistical computing. (R Foundation for Statistical Computing, 2013).

2. Loman, N. J. \& Quinlan, A. R. Poretools: a toolkit for analyzing nanopore sequence data. Bioinformatics btu555- (2014). doi:10.1093/bioinformatics/btu555

3. Huppert, J. L. Structure, location and interactions of G-quadruplexes. FEBS J. 277, 3452-8 (2010).

4. Huppert, J. L. \& Balasubramanian, S. Prevalence of quadruplexes in the human genome. Nucleic Acids Res. 33, 2908-16 (2005).

5. Quinlan, A. R. \& Hall, I. M. BEDTools: a flexible suite of utilities for comparing genomic features. Bioinformatics 26, 841-2 (2010). 